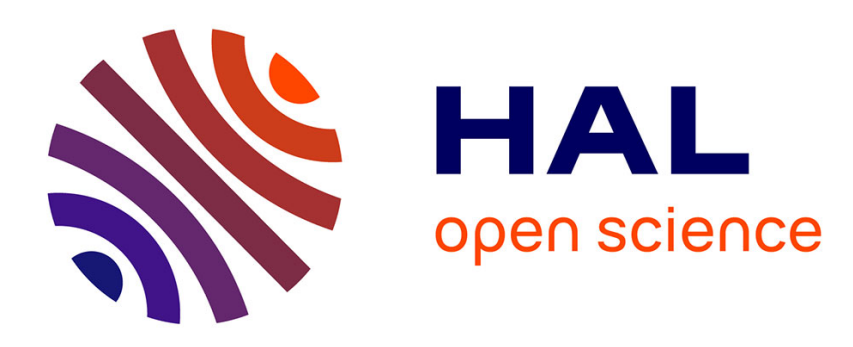

\title{
Estimation of the electrical potential distribution along metallic casing from surface self-potential profile
}

\author{
Alexis Maineult
}

\section{To cite this version:}

Alexis Maineult. Estimation of the electrical potential distribution along metallic casing from surface self-potential profile. Journal of Applied Geophysics, 2016, 10.1016/j.jappgeo.2016.03.038 . hal01300949

\section{HAL Id: hal-01300949 \\ https://hal.sorbonne-universite.fr/hal-01300949}

Submitted on 11 Apr 2016

HAL is a multi-disciplinary open access archive for the deposit and dissemination of scientific research documents, whether they are published or not. The documents may come from teaching and research institutions in France or abroad, or from public or private research centers.
L'archive ouverte pluridisciplinaire HAL, est destinée au dépôt et à la diffusion de documents scientifiques de niveau recherche, publiés ou non, émanant des établissements d'enseignement et de recherche français ou étrangers, des laboratoires publics ou privés. 
Estimation of the electrical potential distribution along metallic casing from surface selfpotential profile

Alexis Maineult

Sorbonne Universités, UPMC Univ Paris 06, CNRS, EPHE, UMR 7619 Metis, 4 place Jussieu, 75005 PARIS, France

E-mail: alexis.maineult@upmc.fr, tel: +33 (0)1 44274336 


\section{ABSTRACT}

Corroding casings of wells generate negative self-potential (SP) anomalies, increasing from about -10 to $-500 \mathrm{mV}$ in the vicinity of the well to $0 \mathrm{mV}$ at large distances. As reported in previous laboratory experiment, SP can be used to retrieve the distribution of electrical potential along the casing, which is somehow a proxy for the corrosion state of the casing. These studies used 3D (whole space) or surface 2D (whole surface) measurements of SP distribution; here we reported a field example, for which only a 1D surface SP profile is available. In order to retrieve the most probable associated potential distribution (defined by a spline) along the 11.1-m long metallic casing, we develop a direct model based on geometrical and geoelectrical properties of the medium, which was then used in a (non-deterministic) optimization procedure by simulated annealing, including some physical constrains. Tests carried out on a synthetic case allowed the initial source to be correctly retrieved, provided that the number of nodes used for the spline defining the potential distribution along the casing is large enough. The inversion of real field data provided a dipolar anomaly, with minimal negative amplitude of around $-600 \mathrm{mV}$ at $5 \mathrm{~m}$, and maximal positive amplitude of about $1100 \mathrm{mV}$ at $9 \mathrm{~m}$ (close to the level of the water table), this shape being in agreement with the results of previous laboratory studies.

Keywords: self-potential, metallic casing, corrosion, simulated annealing 


\section{Introduction}

The self-potential (SP) method, which consists in measuring the natural electrical potential, has been applied for the characterization of fluid circulation in the subsurface since the 1970's. Indeed, due to the electrokinetic effect, the movement of electrolyte through porous media generates a local electric field, generally oriented along the opposite of the flow direction. Recording the electrical potential at the surface therefore provides information about the water movement, such as its direction and amplitude. The method presents the advantage of being lowcost, easy to implement in the field, and to allow the site to be investigated with a dense spatial sampling (and even temporal one if time-lapse measurements are required). The potential difference is simply measured between two unpolarisable electrodes (Petiau and Dupis, 1980; Petiau, 2000) placed at the surface of the ground, possibly with bentonite saturated with brine to ensure a good electrical contact with the soil. The reference electrode is kept fixed far from the considered zone of expected SP anomaly, whereas the measuring electrode is moved to each measurement point, in order to obtain profiles or maps. As the expected values of the potential generally range between 5 and $100 \mathrm{mV}$, the potential difference is measured with an ohm-meter with high entrance impedance.

Numerous studies have been published during the two last decades about the use of the SP method for hydrological purposes (see for instance the reviews by Bolève et al., 2007; Jouniaux et al., 2009; Revil et al., 2012 and references therein). In particular, the SP method gave excellent results when applied to pumping tests. In this case, the SP profiles are anticorrelated with the drawdown, as observed in laboratory experiments (e.g., Suski et al., 2004) or in the field (e.g., Bogoslovsky and Ogilvy, 1973; Rizzo et al., 2004), allowing the aquifer properties, such as transmissivity, to be retrieved using different algorithms (e.g., Patella, 1997; Darnet et al., 2003; Revil et al., 2003; Titov et al., 2005). However, in the field, the electrokinetic signal of interest 
can be masked by additional potentials, in particular when the well casing is metallic. In this case, the casing may act as an electronic conductor between the oxidizing upper part of the subsurface, enriched in oxygen, and the reducing, depleted deeper part. This system, acting as a halfgenerator, is known as the geobattery (Sato and Mooney, 1960; Stoll et al., 1995; Bigalke and Grabner, 1997): the upper part of the metallic conductor plays the role of the cathode, with reduction reactions, and is therefore negatively charged, whereas the lower one is the positively charged anode, with oxidation reactions; electrons flow through the metallic body and outside it ionic currents close the circuit. The resulting anomaly at the surface is negative, with amplitude that can reach more than $500 \mathrm{mV}$. This mechanism has been successfully applied to explain the SP anomaly observed near the KTB deep well and resulting from the existence of graphitic dykes (Bigalke and Grabner, 1997). Moreover, the signal can evolve with time, when corrosion phenomena develop (e.g., Castermant et al., 2008; Rittgers et al., 2013). When measuring SP potential during pumping tests in the case of metallic casing, the anomaly resulting from the fluid flow can be obtained by subtracting the SP values measured before the test (i.e., resulting uniquely from the geobattery effect).

Here we reported a case for which this initial correction cannot be applied. Our primary goal was to evaluate the ability of the SP method to detect and even characterize the fluid circulation in an In-Situ-Recovery (ISR) cell. ISR is a technique used to extract soluble ore by injecting solvent in one or more well and pumping it in recovery wells. Examination of the SP map and profiles evidenced that the predominant component of the signal was due to corrosion effect. But since it is almost impossible to stop an already functioning ISR cell, the correction described above was not applicable. Moreover, even if the production could be stopped, solvents remaining in the medium could have an effect on the SP signal, which will be added to the pure corrosion signal. The problem here was that we have no "zero state" SP signals to perform the 
correction.

However, a piezometer with similar casing is located near the cell, far enough for not being influenced by the fluid circulation. The SP profile shown on Fig. 1 exhibits a negative anomaly, reaching about $-160 \mathrm{mV}$ close to the casing, and returning to zero at around $15 \mathrm{~m}$. The shape is very similar to those reported by Castermant et al. (2008) in a laboratory experiment in which a metallic rod was let free to corrode in a tank filled with saturated sand. The idea was to use the SP profile from the piezometer to correct the observed SP anomaly on the ISR cell. In theory, from previous laboratory experiments, the potential at the surface resulting from the corrosion of the well casing can be modelled (e.g., Castermant et al., 2008), provided that information about the properties of the constituents of the casing and the chemistry of the medium, such as $\mathrm{pH}$ and redox potential distributions, are known. Unfortunately, in the field we did not have this information.

If direct modelling is not possible, inversion of SP can be performed. In the previous laboratory studies published in the literature (Castermant et al., 2008; Rittgers et al., 2013), retrieval of potential along the casing was performed from 3D (whole space) or 2D (whole surface) measurements of SP, using deterministic algorithms. In our case, we only have a 1D surface profile: the problem is largely badly constrained. That is the reason why we develop a non-deterministic inversion procedure to estimate the most probable electrical potential distribution along the well casing. The casing is considered as the unique source of SP signals and no a priori about the underlying chemical phenomena is required. Only the geometry of the system and the distribution of resistivity of the medium that can be obtained easily by welllogging and sampling and/or electrical resistivity tomography, are needed. Then, from the "best" distribution along the well, the whole potential distribution resulting in the medium by diffusion can be inferred using Poisson equation, and therefore the values of the potential at the surface. 
Interestingly, the method also constitutes a good interpolator for the observed data.

\section{Direct problem}

The direct problem consists in solving Poisson equation $\nabla \bullet(\sigma \nabla V)=S$, where $\sigma$ stands for the conductivity distribution, $V$ for the electrical potential, and $S$ for the source term, over the subsurface. From field considerations and well-logging and sampling, we considered a half medium with two layers (i.e., the unsaturated zone and the saturated aquifer), of conductivity $\sigma_{u}=1 / 200 \mathrm{~S} \mathrm{~m}^{-1}$ and $\sigma_{s}=1 / 30 \mathrm{~S} \mathrm{~m}^{-1}$ respectively, the thickness of the upper layer being denoted $W$. The well is $200 \mathrm{~m}$ long, the upper part being a metallic casing with an inner radius $R=19.37$ $\mathrm{cm}$, a length $L=11.1 \mathrm{~m}$, and a wall thickness $t=7 \mathrm{~mm}$. The lower casing of the well is made of PVC and is thus impervious and insulating. We consider the whole aquifer unconfined and at equilibrium, so the water levels in the borehole and in the medium are equal. Inside the well, the water has a conductivity $\sigma_{f}=1 / 5 \mathrm{~S} \mathrm{~m}^{-1}$ (Fig. 2). For sake of simplicity, we will consider it constant with depth.

The domain is thus divided into 5 sub-domains, denoted (1) (the unsaturated zone outside the well), (2) and (2') (the saturated zone outside the well), and (3) and (3') (the free water inside the well). Poisson equation can be simplified as:

$$
\Delta V(r, z)=\frac{\partial^{2} V(r, z)}{\partial r^{2}}+\frac{1}{r} \frac{\partial V(r, z)}{\partial r}+\frac{\partial^{2} V(r, z)}{\partial z^{2}}=0
$$

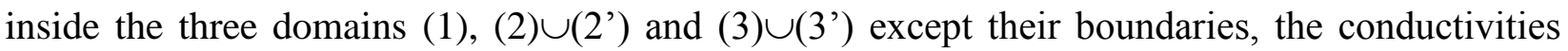
being constant inside each domain. The boundaries conditions are:

- no-flux at the surface:

$$
\forall r \in\left[0, R\left[\frac{\partial V(r, z)}{\partial z}\right)_{z=W}=0\right.
$$


$\forall r \in] R+t,+\infty\left[\frac{\partial V(r, z)}{\partial z}\right)_{z=0}=0$

- the potential is equal to zero at infinity:

$\forall z \in\left[0,+\infty\left[\quad \lim _{r \rightarrow+\infty} V(r, z)=0\right.\right.$

$\forall r \in\left[0,+\infty\left[\lim _{z \rightarrow+\infty} V(r, z)=0\right.\right.$,

- along the axis $r=0$, the first derivative of $V$ with respect to $r$ is equal to zero for symmetry reasons:

$\forall z \in\left[W,+\infty\left[\frac{\partial V(r, z)}{\partial r}\right)_{r=0}=0\right.$

- along the metallic casing, the potential is fixed:

$\forall z \in\left[0, L_{m}\right] \quad V(R, z)=V(R+t, z)=f(z)$

- along the PVC casing (insulating), there is no normal flux:

$\left.\forall(r, z) \in\{R, R+t\} \times] L_{m}, L_{m}+L_{p}\right] \quad \frac{\partial V(r, z)}{\partial \mathbf{n}}=0$

where $\mathbf{n}$ is the unit vector normal to the well.

- at the interface between sub-domains (1) and (2), Poisson equation writes:

$\Delta V(r, z)=\frac{\partial^{2} V(r, z)}{\partial r^{2}}+\frac{1}{r} \frac{\partial V(r, z)}{\partial r}+\frac{\partial^{2} V(r, z)}{\partial z^{2}}+\frac{\partial \ln \sigma(r, z)}{\partial z} \frac{\partial V(r, z)}{\partial z}=0$

We solved Poisson equation in cylindrical coordinates using the centred finite-difference scheme, on a grid based on the simplified geometry of the subsurface (Fig. 3). The mesh size is geometrically increasing towards infinity, except for the radial distance $r$ comprised between 0 and $R$ and for the depth $z$ between 0 and $L_{m}$ where the spacing is constant. Note that the first mesh in the $z$-direction and the first mesh in the $r$-direction were subdivided with a geometrically 
increasing spacing, in order to make the value of the potential near the surface and near the well more accurate. From tests (see below), the limits of the mesh were fixed to $r=Z=10 \mathrm{~km}$, even though the domain of real interest is restricted to $(r, z) \in\left[\begin{array}{ll}0 & 50\end{array}\right] \times\left[\begin{array}{ll}0 & 50\end{array}\right] \mathrm{m}$. The total number of nodes was $\mathrm{I} \times \mathrm{J}=64 \times 71$, and so the total number of cells was 4410 .

Discretization procedure and discretized equations are given in Annex 1. The discretized system can write $\mathbf{M V}=\mathrm{S}$, where $\mathbf{M}$ is a sparse, square matrix of dimension I.J $\times \mathrm{I} . \mathrm{J}, \mathrm{V}$ the vector of the potential at the nodes, and $\mathrm{S}$ a source term. For a given distribution of potential along the metallic casing, the potential distribution is thus given by:

$\mathrm{V}=\mathbf{M}^{-1} \mathbf{S}$

To check the efficiency of the discretization (i.e., irregular, rectangular mesh), the system was modified to compute the response of the uniformly charged hollow cylinder buried in a homogeneous half-space. The numerical predictions compare well with the analytical solution (see Annexes 2 and 3).

An example of application is shown in Fig. 4. Note that for practical reasons, we do not directly fix the potential value at each grid node of the metallic casing, but instead we fixed the potential values at some chosen grid nodes of the casing, and interpolate these values at all the grid nodes of the casing, using a spline. In this way, the number of parameters to be inverted during the inverse problem is reduced. From the given potential distribution on the metallic casing (positions of spline nodes: 0, 1, 4.75, 9.5 and $11.1 \mathrm{~m}$, associated potential values: -200, 210, $-600,800$ and $0 \mathrm{mV}$ respectively, Fig. 4a), the distribution of potential over the whole grid is calculated using Eq. (8) (Fig. 4b). The surface profile is then extracted (Fig. 4c).

\section{Inverse problem: simulated annealing}


The inverse problem consists in retrieving the distribution of potential along the metallic casing from the surface profile, i.e., to find the values of the potential at the node of the spline, the position of which being fixed a priori. To explore the space of the possibles sources (S-term in Eq. (8)), we chose to use the simulated annealing algorithm

Simulated annealing, or statistical cooling, is a Monte-Carlo iterative improvement method. It allows the stepping through the parameter space (here all the possible sources S) towards the subset that minimizes the cost-function (i.e., an evaluation of the difference between the data and the predictions). The way of going down towards the optimal configuration is controlled by the temperature, a parameter that decreases in order to eliminate the models with the largest cost-function progressively. A very interesting characteristic of this algorithm is that it presents the ability to escape from local minima by accepting new configurations of the parameter space with larger cost values. Here we adopted the formalism developed by Aarts and Van Laarhoven (1985).

The algorithm starts from an initial model $\mathrm{S}$, with an associated value $F(\mathrm{~S})$ of the costfunction, and an initial temperature $T$ (Fig. 5). Then a new model $\mathrm{S}^{\prime}$ is generated by perturbing the initial model $\mathrm{S}$, and the associated cost-function value $F\left(\mathrm{~S}^{\prime}\right)$ is computed. An acceptance test is carried out. If the new model is accepted, a test is performed to check if the predicted data are monotonously increasing. If the monotony test is positive, the new model is accepted, i.e., the current model is now $\mathrm{S}=\mathrm{S}$ '. If this model has a lower cost-function than the current optimal solution $\mathrm{S}_{\text {opt }}$, then the optimal solution is updated, i.e., $\mathrm{S}_{\text {opt }}=\mathrm{S}$. If the acceptance test or the monotony test is negative, then the new model is rejected, and the current model remains equal to S. Afterwards, a stabilisation test is carried out. If it is negative, a new model is generated as before, until the stabilization test becomes positive. This series of loops at constant temperature constitutes what we called iteration. When the stabilization test is positive, a stop test is 
performed. If it is positive, the algorithm ends; if it is negative, the temperature is lowered, and a new iteration starts, with $\mathrm{S}_{\text {opt }}$ as a starting model.

Generally, we started an initial inversion with the initial model being $\{0\}_{1, \ldots, N}$, where $N$ is the size of S, and allowed large amplitude for the perturbations of the model, in order to explore the whole parameter space roughly. The optimal solution so produced was used as starting model for a second inversion, with smaller amplitude of the model perturbations, in order to refine the search around the optimal solution. This last step was repeated until the final optimal solution remains equal to the initial model, i.e., no further improvement of the solution could be made.

\section{Perturbation of the current model}

The current model $\mathrm{S}$ can be written as $\left\{S_{i}\left(j_{i}\right)\right\}, i=\{1, \ldots, N\}$, since each component $S_{i}$ is allowed to take discrete values in an interval $\left\{S_{i, \min }, \ldots, S_{i, \max }\right\}$. For each component, an entire $n_{i}$ is randomly generated using a Gaussian distribution with a mean of 0 and a chosen standard deviation $e$. Then the new model is given by:

$$
\left\{S_{i}^{\prime}\left(j_{i}^{\prime}\right)\right\}=\left\{S_{i}\left(j_{i}+n_{i}\right)\right\} \text {. }
$$

The parameter $e$ fixed the amplitude of the allowed perturbation.

\section{Cost-function}

The cost function $F(\mathrm{~S})$ is an estimation of the fit between the data $\mathrm{D}^{o b s}=\left\{D_{j}^{o b s}\right\}$, $j=\left\{1, \ldots, N_{d}\right\}$ (i.e., synthetic or observed surface SP profile) and the results from the model S,

denoted $\mathrm{D}^{\text {pred }}=\left\{D_{j}^{\text {pred }}\right\}$ (predicted surface profile), where $N_{d}$ is the size of the data vector. We chose to apply the $\mathrm{L}_{1}$-norm: 


$$
F(\mathrm{~S})=\left\|\mathrm{D}^{\text {pred }}-\mathrm{D}^{o b s}\right\|_{1}=\frac{1}{N_{d a t}} \sum_{j}^{N_{d}}\left|D_{j}^{\text {pred }}-D_{j}^{o b s}\right| .
$$

The use of the L1-norm provides much better convergence than the more classical $\mathrm{L}_{2}$-norm.

\section{Acceptance test}

The acceptance probability of the new model is defined by:

$$
A=\min \left(\exp \left(-\frac{F\left(\mathrm{~S}^{\prime}\right)-F(\mathrm{~S})}{T}\right), 1\right)
$$

If a value randomly generated in $[0,1]$ (using a uniform distribution) is less or equal to $A$, the new model S' is accepted as new current model, otherwise it is rejected. In other words, if the new model $S^{\prime}$ is better than the current model S (i.e., lower value of its cost-function), the acceptance probability is equal to 1 . But new model with higher value of the cost-function may be accepted too, thus allowing escaping from local minima. The temperature $\mathrm{T}$ rules the probability of such acceptance. Low temperature reduces the probability for a worst new model to be accepted.

\section{Monotony test}

Accepted solution can produces surface SP profiles $\mathrm{D}^{\text {pred }}$ that are not monotonously increasing. As this behaviour was not observed in our data (Fig. 1), we add a supplemental test to reject this kind of solutions. Note that if this test is not performed, the algorithm converges but in a slower way.

\section{Stabilization test}

As explained before, an iteration consists of series of loops at a given temperature. One important question is to decide when stopping the iteration and decreasing the temperature. 
Indeed, an early stop can prevent the parameter space from being fully sampled, whereas a late stop can yield to a redundant exploration, so wasting computation time. We use the evolution of the deviation of the cost-function values. This deviation $d(l)$ after the $l^{\text {th }}$ loop is given by:

$d(l)=\sqrt{\left|\frac{1}{l} \sum_{k=1}^{l} F\left(\mathrm{~S}^{k}\right)^{2}-\left(\frac{1}{l} \sum_{k=1}^{l} F\left(\mathrm{~S}^{k}\right)\right)^{2}\right|}$

where the $S^{k}, k=\{1, \ldots, l\}$, are the $l$ models generated on the $l$ previous loops. At the beginning of the iteration, the deviation $d$ varies a lot, but it tends to stabilized with increasing $l$. To estimate the quality of the stabilization, we use the criterion:

$h\left(d, l, w_{h}\right)=\frac{\operatorname{std}\left(\{d(k)\}_{k=l-w_{h}+1, l}\right)}{\left\langle\{d(k)\}_{k=l-w_{h}+1, l}\right\rangle}=\frac{\sqrt{\frac{1}{w_{h}-1} \sum_{k=l-w_{h}+1}^{l}\left|d(k)-\frac{1}{w_{h}} \sum_{k=l-w_{h}+1}^{l} d(k)\right|^{2}}}{\frac{1}{w_{h}} \sum_{k=l-w_{h}+1}^{l} d(k)}$.

It estimates the heterogeneity of the deviation $d$ over the last $w_{h}$ loops. When $h$ is low, the value of $d$ is stabilized and the also the variations around it values.

As soon as $h$ is below a given value $h_{c, d}$, the iteration is stopped. The number of loops carried out depends on the $h_{c, d}$ value. High values of $h_{c, d}$ result in a small number of generated model. It is important to note that in our algorithm the number of model per iterations is not fixed. It only depends on the $h$ values, and therefore from the way the exploration occurs during the iteration.

\section{Stop criterion}

The same question occurs concerning the stop of the algorithm. We considered the rejection rate $r$, i.e. the number of rejected models divided by the number of generated models. This quantity tends towards 1 as so temperature decreases towards 0 , since in this case the 
acceptance probability tends also towards 0 . The heterogeneity of $r$ is thus a good estimate of the stabilization of the algorithm. It is computed as $h\left(r, i, w_{H}\right)$, where $i$ is the number of the current iteration. Again, a low value of $\mathrm{h}$ indicates a stabilization of $r$; the algorithm is stopped when it is below a given value $h_{c, r}$.

\section{Temperature decrease}

The decrease in temperature depends on the deviation of the cost-function of the $N_{i}$ models generated during the iteration. It is given by (Aarts and Van Laarhoven, 1985):

$$
T^{\prime}=T\left[1+\frac{\frac{T}{3} \ln (1+v)}{\sqrt{\frac{1}{N_{i}} \sum_{k=1}^{N_{i}} F\left(\mathrm{~S}_{k}\right)^{2}-\left(\frac{1}{N_{i}} \sum_{k=1}^{N_{i}} F\left(\mathrm{~S}_{k}\right)\right)^{2}}}\right]^{-1}
$$

where $v$ is a parameter equal to 0.1 . The temperature will decrease significantly if the deviation of the cost-function values is small, but will remains close to its previous value if the deviation is high, i.e., if the convergence towards a solution is not satisfactorily.

\section{Example}

An example of parameters output is shown in Fig. 6, for the inversion of the synthetic data presented on Fig. 4c, using a spline with six node (i.e., $N=6$ ) positioned linearly with depth. The initial model is zero potential values for each spline node. The last node was fixed to 0 during the inversion. The possible values for the spline nodes are $\{-2000,-1999, \ldots, 1999,2000\} \mathrm{mV}$, and the standard-deviation of the Gaussian distribution for the perturbation is equal to 5. The initial temperature is equal to 1 , and the stabilization and stop criterions $h_{c, d}$ and $h_{c, r}$ to 0.05 and 0.01 respectively, with $w_{h}=w_{H}=50$. In this case, the number of iterations is equal to 219 . The 
temperature decreases regularly (Fig. 6c). The cost-function of the global optimal solution $\mathrm{S}_{\text {opt }}$ (red line in Fig. 6b) decreases drastically during the first 50 iterations, and then more slowly, whereas the maximal value of the cost-function for the accepted models decreases regularly (purple line in Fig. 6b), as expected from the decrease of the acceptance probability. The number of models generated per iteration varies between 100 and more than 400 (Fig. 6c), and the rejection rate (Fig. 6d) tends towards 1, reaching a plateau after 200 iterations. We can note that the algorithm stopped before the cost-function of the global optimal solution was stabilized.

As explained before, we carried out a second inversion after the initial one, starting from its global optimal solution, in order to refine the search in its vicinity, and be sure that the solution was well stabilized. An example of the interest of this procedure, as well as the criteria for estimating that the solution is stabilized, is given in Fig. 7. In Fig. 7a, we can see the evolution of the potential value at a node, for an initial inversion starting from 0 (the data are the real one (Fig. 1), using a spline with 6 nodes linearly positioned with depth, the last one being kept fixed; the presented node is node $\mathrm{n}^{\circ} 4$; inversions parameters are the same as before). The potential value at the node varies between -200 and $400 \mathrm{mV}$ during the initial phase of exploration, but seems to be stabilized at $108 \mathrm{mV}$ after 100 iterations. Examination of the values of the potential at the node as a function of the values of the associated cost-function (Fig.s 7b and c) shows that there are in fact two set of close solutions, one around $100 \mathrm{mV}$ and the others close to $350 \mathrm{mV}$. When plotting the distribution of the values of the potential at the node for costfunction values less than $2.2 \mathrm{mV}$, we see that the optimal value determined by the inversion, and the average and median values of the distribution are not equal. A second inversion, then a third inversion starting from the result of the second one (modified inversion parameters: initial temperature 0.1 , standard deviation for the perturbation of $1, h_{c, l}=0.025$ and $h_{c, r}=0.005$ ) have been carried out to refine this first exploration. The third iteration shows a complete stabilization of the 
potential value, around $-222 \mathrm{mV}$, a value very different from the value determined during the initial inversion (Fig. 7e). In this case, the optimal value, the average value and the median value of the models with lower cost-functions are quasi equal, making us considering that the optimal solution is reached.

Note that in the following we will call full inversion the set of inversions necessary to obtained a stabilized solution

\section{Applications}

\subsection{Synthetic case}

It is important to note that the position vector of the spline nodes with depth is not known a priori, and it thus constitutes an input parameter of the inverse problem. We first studied the effect of the number of nodes used, i.e. from 4 to 7 nodes, linearly positioned with depth. To do so, we invert the synthetic data shown in Fig. 4c, and then compared the retrieved potential distribution with the true source (Fig. 4a). In a first stage, in order to reduce the parameter space size and to save computational times, we fixed the potential value of the deepest node to its solution, i.e., to zero. Note that this constrain can have a physical meaning: if there is continuity, the potential at the connection between the metallic and the PVC casings should be null, the PVC casing being not charged since it is an insulator. The retrieved sources are similar to the input one, except the 4-node solution (Fig. 8a). The predicted data for splines with 5, 6 and 7 nodes are extremely similar and explain very well the synthetic data, with $\mathrm{L}_{1}$-norm (cost-function values for the inverse problem) less than $0.077 \mathrm{mV}$, and adjustment coefficient $r^{2}$ equal to 1 (Fig. $8 \mathrm{~b}$ ). The classical $\mathrm{L}_{2}$-norm, or Root Mean Square (RMS), is even better for the splines with 6 and 7 nodes (less than $0.01 \mathrm{mV}$ ). When comparing the retrieved sources, only solution with 6 and 7 nodes are acceptable (Fig. 8a). The best adjustment (calculated using the values of the potential interpolated 
by the spline at the grid node along the casing) is obtained for the spline with 6 nodes $\left(\mathrm{L}_{1}\right.$-norm equal to $5.1 \mathrm{mV}, \mathrm{L}_{2}$-norm to $7.1 \mathrm{mV}$, adjustment coefficient to 1). In this case, it seems therefore that 6 nodes, linearly positioned with depth, are sufficient to retrieve the source.

We then studied the effect of fixing the potential value of the deepest node to 0 on the solution. Starting from the previous solutions, we carried out a new full inversion, but letting the deepest node free to move. In this case, the predicted data (not shown here), are very similar to those obtained previously (Fig. 8b). Splines with 4 and 5 nodes changed a lot (Fig. 9); the potential value of the deepest node for the 4-node spline even diverged. But splines with 6 and 7 did not changed significantly ( -6 and $-2 \mathrm{mV}$ for the deepest node, instead of 0 , which is the true value). Here again, the best source correspond to the 6-node spline.

We also studied the effect of noise. To do so, we again invert the synthetic data (Fig. 4c), but with added Gaussian noise (mean $0 \mathrm{mV}$ and standard-deviation of $2 \mathrm{mV}$ ). To exclude the influence of the node positions, we used the true node positions (input for the computation of the synthetic data, i.e., $0,1,4.75,9.5$ and $11.1 \mathrm{~m})$. We can note here that when using these node positions and without noise, the true source is perfectly retrieved. We can see that the noise disturb the solution (Fig. 10), but the global shape is preserved. This point is important, knowing that the noise on real data carefully acquired is generally in the range $\pm 2 \mathrm{mV}$.

\subsection{Real case}

Finally, we applied the process to the real data (Fig. 1). The nodes were linearly positioned with depth, and the inversion procedure was carried in two steps. A first full inversion was carried out with the potential value of the deepest node fixed to 0 ; a second full inversion then followed, with this potential value free to move, starting from the solution of the first full 
inversion. In this case, the spline with 4 node diverges again, but the splines with 5, 6 and 7 nodes exhibit similar features, i.e., a dipolar aspect with a negative pole between 4 and $5 \mathrm{~m}$ with an amplitude of $-600 \mathrm{mV}$ and a positive one between 9 and $10 \mathrm{~m}$ with an amplitude of about 1100 $\mathrm{mV}$ (Fig. 11a); all solutions explain very well the data (Fig. 11b), with L1-norm around 2 and adjustment coefficient close to 1 . The retrieved distribution of potential in the whole space (Fig. 12) compared in a very satisfactorily way to the observations made by Rittgers et al. (2013) during the corrosion of a small vertical metallic hollow cylinder in a sandbox connecting a saturated and a unsaturated zone in a metric sandbox, being negative in the upper zone, and positive in the lower one. This coherent result made us very confident with our inversion procedure.

\section{Conclusions}

Based on hydrological and logging information about the medium (i.e., water table depth, fluid conductivity, saturated and unsaturated sand conductivities), and on geometrical characteristics of the well, we solved Poisson equation in order to compute the electrical potential in the medium resulting from an imposed potential distribution along the casing.

This direct problem was integrated in an optimization procedure by simulated annealing in order to retrieve the most probable distribution along the metallic casing from surface profile. The algorithm converges towards a solution with physical meaning provided that the number of nodes chosen for the spline defining the potential distribution along the casing is sufficient on the one hand, and that the noise on the data does not exceed few $\mathrm{mV}$ (for a surface anomaly of 200 $\mathrm{mV}$ of maximum amplitude) on the other hand.

Applied to a real case of corroding metallic casing, for which a self-potential profile was acquired at the surface, the algorithm provided a dipolar distribution of the potential along this 
casing, with a negative pole in the unsaturated zone. This distribution is very similar to the laboratory results obtained by Castermant et al. (2008) and Rittgers et al. (2013), making us confident in the efficiency of our algorithm. Note here that we introduce a non-deterministic approach for using SP surface profiles (largely badly constrained problem), contrary to Rittgers et al. (2013) who used deterministic approach for 3D SP measurements in the whole space (relatively well constrained problem), allowing us to establish a statistics on the possible potential distribution.

A first application may be to use this procedure as a physics-based interpolator for such kind of data (see Fig. 11b). Second, it may be a tool to evaluate the evolution of the corrosion of metallic casings from surface self-potential measurements - as also suggested by Rittgers et al. (2013) - i.e., without using logging tools (and therefore without stopping the production, if any) thus saving time and money.

Finally, an interesting implementation, beyond the scope of this paper, may be to integrate a procedure to evaluate the distribution of redox potential associated with the retrieved electrical potential source, as done by Rittgers et al. (2013) for instance.

\section{Acknowledgments}

The author thanks AREVA Mines for funding the field campaign and some preliminary works. The author also thanks Elodie Williard, Mickaël Béhaegel, Jean-Marc Miehé and Valérie Langlais from AREVA Mines, and Anatole Bruhat.

\section{Annex 1. Discretization}

Using the discretization scheme (with irregular spacing) shown in Fig. A1, we can deduce the approximation of the first derivative of $V$ in $x_{i}$ from Taylor expansions of the function $V$ in the 
vicinity of $x_{i}+d x$ and $x_{i}-d x$ :

$$
\left.\frac{d V}{d x}\right)_{x_{i}}=\frac{V_{i+1}}{2 d x_{i}}+\frac{1}{2}\left(\frac{1}{d x_{i-1}}-\frac{1}{d x_{i}}\right) V_{i}-\frac{V_{i-1}}{2 d x_{i-1}}
$$

Similarly, considering the Taylor expansion of the first derivative of $V$ in the vicinity of virtual points $x_{i-1 / 2}$ and $x_{i+1 / 2}$, we obtain the approximation of the second derivative:

$$
\frac{d^{2} U}{d x^{2}}=\frac{1}{2 d x_{i}}\left(\frac{1}{d x_{i}}+\frac{1}{d x_{i-1}}\right) V_{i+1}-\frac{1}{2}\left(\frac{1}{d x_{i}}+\frac{1}{d x_{i-1}}\right)^{2} V_{i}+\frac{1}{2 d x_{i-1}}\left(\frac{1}{d x_{i-1}}+\frac{1}{d x_{i}}\right) V_{i-1} .
$$

Introducing Eqs. (A1) and (A2) in Eq. (1) gives the equation for the internal domain without the internal interface between domains (1) and (2) and without the well:

$$
\begin{aligned}
& \frac{1}{d z_{j-1}}\left(\frac{1}{d z_{j-1}}+\frac{1}{d z_{j}}\right) V_{i, j-1}+\frac{1}{d r_{i-1}}\left[\left(\frac{1}{d r_{i-1}}+\frac{1}{d r_{i}}\right)-\frac{1}{r_{i}}\right] V_{i-1, j} \\
& +\left[-\left(\frac{1}{d r_{i-1}}+\frac{1}{d r_{i}}\right)^{2}+\frac{1}{r_{i}}\left(\frac{1}{d r_{i-1}}-\frac{1}{d r_{i}}\right)-\left(\frac{1}{d z_{j-1}}+\frac{1}{d z_{j}}\right)^{2}\right] V_{i, j} \\
& +\frac{1}{d r_{i}}\left[\left(\frac{1}{d r_{i-1}}+\frac{1}{d r_{i}}\right)+\frac{1}{r_{i}}\right] V_{i+1, j}+\frac{1}{d z_{j}}\left(\frac{1}{d z_{j-1}}+\frac{1}{d z_{j}}\right) V_{i, j+1}=0
\end{aligned}
$$

where $d r_{i}$ and $d z_{j}$ are the dimension of mesh cell $(i, j),(i, j)$ being the indexes of the upper left corner of the cell. Concerning the interface, the $\sigma$-term in Eq. (7) becomes:

$$
\frac{\partial \ln \sigma(r, z)}{\partial z} \frac{\partial V(r, z)}{\partial z}=\frac{\ln \frac{\sigma_{j}}{\sigma_{j-1}}}{4 d z_{j-1}}\left(\frac{1}{d z_{j}} V_{i, j+1}+\left(\frac{1}{d z_{j-1}}-\frac{1}{d z_{j}}\right) V_{i, j}-\frac{1}{d z_{j-1}} V_{i, j-1}\right)
$$

Thus at the internal interface between media (1) and (2) we must use: 


$$
\begin{aligned}
& \left(\frac{1}{d z_{j-1}}\left(\frac{1}{d z_{j-1}}+\frac{1}{d z_{j}}\right)-\frac{1}{2 d z_{j-1}{ }^{2}} \ln \frac{\sigma_{j}}{\sigma_{j-1}}\right) V_{i, j-1}+\frac{1}{d r_{i-1}}\left[\left(\frac{1}{d r_{i-1}}+\frac{1}{d r_{i}}\right)-\frac{1}{r_{i}}\right] V_{i-1, j} \\
& \quad+\left[-\left(\frac{1}{d r_{i-1}}+\frac{1}{d r_{i}}\right)^{2}+\frac{1}{r_{i}}\left(\frac{1}{d r_{i-1}}-\frac{1}{d r_{i}}\right)-\left(\frac{1}{d z_{j-1}}+\frac{1}{d z_{j}}\right)^{2}+\frac{1}{2 d z_{j-1}}\left(\frac{1}{d z_{j-1}}-\frac{1}{d z_{j}}\right) \ln \frac{\sigma_{j}}{\sigma_{j-1}}\right] V_{i, j} \\
& \quad+\frac{1}{d r_{i}}\left[\left(\frac{1}{d r_{i-1}}+\frac{1}{d r_{i}}\right)+\frac{1}{r_{i}}\right] V_{i+1, j}+\left(\frac{1}{d z_{j}}\left(\frac{1}{d z_{j-1}}+\frac{1}{d z_{j}}\right)+\frac{1}{2 d z_{j-1} d z_{j}} \ln \frac{\sigma_{j}}{\sigma_{j-1}}\right) V_{i, j+1}=0
\end{aligned}
$$

instead of Eq. (A3).

To discretize the boundary conditions, we introduce a virtual shelf as:

1) $\quad V_{i, j-1}=V_{i, j}$ and $d z_{j-1}=d z_{j}$ at the surface (i.e., $j=j_{W}$ inside the well and $j=1$ outside) to take into account Eqs. (2),

2) $\quad V_{i+1, j}=0$ and $d r_{i}=d r_{i-1}$ for $r$ infinite (i.e., $i=I$ ), to take into account Eq. (3a),

3) $\quad V_{i, j+1}=0$ and $d z_{j}=d z_{j-1}$ for $z$ infinite (i.e., $j=J$ ), to take into account Eq. (3b),

4) $\quad V_{i-1, j}=V_{i+1}, j$ and $d r_{i-1}=d r_{i}$ for $r=0$ (i.e., $i=1$ ), to take into account Eq. (4).

The equations for the external boundaries without their corners are:

- at the surface inside the well, without considering $r=0$ (Eq. (2a) into Eq. (1)):

$$
\begin{gathered}
\frac{1}{d r_{i-1}}\left[\left(\frac{1}{d r_{i-1}}+\frac{1}{d r_{i}}\right)-\frac{1}{r_{i}}\right] V_{i-1, j}+\left[-\left(\frac{1}{d r_{i-1}}+\frac{1}{d r_{i}}\right)^{2}+\frac{1}{r_{i}}\left(\frac{1}{d r_{i-1}}-\frac{1}{d r_{i}}\right)-\frac{2}{d z_{j}^{2}}\right] V_{i, j} \\
+\frac{1}{d r_{i}}\left[\left(\frac{1}{d r_{i-1}}+\frac{1}{d r_{i}}\right)+\frac{1}{r_{i}}\right] V_{i+1, j}+\frac{2}{d z_{j}^{2}} V_{i, j+1}=0
\end{gathered}
$$

- at the surface outside the well for $r$ not equal to infinity (Eq. (2b) into Eq. (1)): 


$$
\begin{aligned}
\frac{1}{d r_{i-1}} & {\left[\left(\frac{1}{d r_{i-1}}+\frac{1}{d r_{i}}\right)-\frac{1}{r_{i}}\right] V_{i-1, j}+\left[-\left(\frac{1}{d r_{i-1}}+\frac{1}{d r_{i}}\right)^{2}+\frac{1}{r_{i}}\left(\frac{1}{d r_{i-1}}-\frac{1}{d r_{i}}\right)-\frac{2}{d z_{j}^{2}}\right] V_{i, j}, } \\
& +\frac{1}{d r_{i}}\left[\left(\frac{1}{d r_{i-1}}+\frac{1}{d r_{i}}\right)+\frac{1}{r_{i}}\right] V_{i+1, j}+\frac{2}{d z_{j}^{2}} V_{i, j+1}=0
\end{aligned}
$$

- in $r$ equal to infinity, without considering $z=0, z$ equal to infinity nor the interface (Eq. (3a) into Eq. (1)):

$$
\begin{aligned}
& \frac{1}{d z_{j-1}}\left(\frac{1}{d z_{j-1}}+\frac{1}{d z_{j}}\right) V_{i, j-1}+\frac{1}{d r_{i-1}}\left[\frac{2}{d r_{i-1}}-\frac{1}{r_{i}}\right] V_{i-1, j}+\left[-\frac{4}{d r_{i-1}^{2}}-\left(\frac{1}{d z_{j-1}}+\frac{1}{d z_{j}}\right)^{2}\right] V_{i, j}, \\
& \quad+\frac{1}{d z_{j}}\left(\frac{1}{d z_{j-1}}+\frac{1}{d z_{j}}\right) V_{i, j+1}=0
\end{aligned}
$$

- in $r$ equal to infinity, at the interface (Eq. (3a) into Eq. (A5)):

$$
\begin{aligned}
& \left(\frac{1}{d z_{j-1}}\left(\frac{1}{d z_{j-1}}+\frac{1}{d z_{j}}\right)-\frac{1}{2 d z_{j-1}^{2}} \ln \frac{\sigma_{j}}{\sigma_{j-1}}\right) V_{i, j-1}+\frac{1}{d r_{i-1}}\left[\frac{2}{d r_{i-1}}-\frac{1}{r_{i}}\right] V_{i-1, j} \\
& \quad+\left(-\frac{4}{d r_{i-1}^{2}}-\left(\frac{1}{d z_{j-1}}+\frac{1}{d z_{j}}\right)+\frac{1}{2 d z_{j-1}}\left(\frac{1}{d z_{j-1}}-\frac{1}{d z_{j}}\right) \ln \frac{\sigma_{j}}{\sigma_{j-1}}\right] V_{i, j}, \\
& \quad+\left(\frac{1}{d z_{j}}\left(\frac{1}{d z_{j-1}}+\frac{1}{d z_{j}}\right)+\frac{1}{2 d z_{j-1} d z_{j}} \ln \frac{\sigma_{j}}{\sigma_{j-1}}\right) V_{i, j+1}=0
\end{aligned}
$$

- in $z$ equal to infinity, without considering $r=0$ nor $r$ equal to infinity (Eq. (3b) into Eq. (1)):

$$
\begin{aligned}
& \frac{2}{d z_{j-1}^{2}} V_{i, j-1}+\frac{1}{d r_{i-1}}\left[\left(\frac{1}{d r_{i-1}}+\frac{1}{d r_{i}}\right)-\frac{1}{r_{i}}\right] V_{i-1, j} \\
& +\left[-\left(\frac{1}{d r_{i-1}}+\frac{1}{d r_{i}}\right)^{2}+\frac{1}{r_{i}}\left(\frac{1}{d r_{i-1}}-\frac{1}{d r_{i}}\right)-\frac{4}{d z_{j-1}^{2}}\right] V_{i, j}+\frac{1}{d r_{i}}\left[\left(\frac{1}{d r_{i-1}}+\frac{1}{d r_{i}}\right)+\frac{1}{r_{i}}\right] V_{i+1, j}=0
\end{aligned}
$$

- in $r$ equal to 0, without considering $z=0$ nor $z$ equal to infinity, (Eq. (4) into Eq. (1)): 


$$
\begin{aligned}
& \frac{1}{d z_{j-1}}\left(\frac{1}{d z_{j-1}}+\frac{1}{d z_{j}}\right) V_{i, j-1}+\left[-\frac{4}{d r_{i}^{2}}-\left(\frac{1}{d z_{j-1}}+\frac{1}{d z_{j}}\right)^{2}\right] V_{i, j}+\frac{4}{d r_{i}{ }_{i}} V_{i+1, j} . \\
& \quad+\frac{1}{d z_{j}}\left(\frac{1}{d z_{j-1}}+\frac{1}{d z_{j}}\right) V_{i, j+1}=0
\end{aligned}
$$

For the four corners, equations are given by:

- at the surface for $r$ infinite (Eqs. (2b) and (3a) into Eq. (1):

$$
\frac{1}{d r_{i-1}}\left[\frac{2}{d r_{i-1}}-\frac{1}{r_{i}}\right] V_{i-1, j}+\left[-\frac{4}{d r_{i-1}^{2}}-\frac{2}{d z_{j}^{2}}\right] V_{i, j}+\frac{2}{d z_{j}^{2}} V_{i, j+1}=0,
$$

- in $r$ and $z$ infinite (Eqs. (3a) and (3b) into Eq. (1)):

$\frac{2}{d z_{j-1}^{2}} V_{i, j-1}+\frac{1}{d r_{i-1}}\left[\frac{2}{d r_{i-1}}-\frac{1}{r_{i}}\right] V_{i-1, j}+\left[-\frac{4}{d r_{i-1}^{2}}-\frac{4}{d z_{j-1}^{2}}\right] V_{i, j}=0$,

- in $r$ equal to 0 and $z$ infinite (Eqs. (4) and (3b) into Eq. (1)):

$\frac{2}{d z_{j-1}^{2}} V_{i, j-1}-\left[\frac{4}{d r^{2}{ }_{i}}+\frac{4}{d z_{j-1}^{2}}\right] V_{i, j}+\frac{4}{d r_{i}^{2}} V_{i+1, j}=0$,

- at the surface in $r$ equal to 0 (Eqs. (2a) and (4) into Eq. (1)):

$$
\left[-\frac{4}{d r_{i}^{2}}-\frac{2}{d z_{j}^{2}}\right] V_{i, j}+\frac{4}{d r_{i}^{2}} V_{i+1, j}+\frac{2}{d z_{j}^{2}} V_{i, j+1}=0 .
$$

For the metallic casing, Eq. (5) yields to:

$$
V_{i, j}=f_{j}
$$

Finally, for the PVC casing, Eq. (6) gives:

- internal wall, without the deepest corner (horizontal flux equal to 0): 
$V_{i-1, j}-V_{i, j}=0$,

- external wall, without the deepest corner (horizontal flux equal to 0):

$V_{i, j}-V_{i+1, j}=0$,

- internal wall, deepest corner (horizontal and vertical fluxes equal to 0):

$V_{i-1, j}-2 V_{i, j}+V_{j+1, i}=0$,

- external wall, deepest corner (horizontal and vertical fluxes equal to 0 ):

$-2 V_{i, j}+V_{i+1, j}+V_{j+1, i}=0$.

Eqs. (A3) and (A5) to (A20) forms the system to be solved.

\section{Annex 2. Analytical solution for the uniformly charges, hollow cylinder buried in a}

\section{homogeneous space}

Let us first consider a uniformly charged hollow cylinder, of radius $a$ and length $L$, in the void (Fig. A2). The potential distribution in the whole space is given by:

$$
\begin{aligned}
V(r, z) & =\frac{1}{4 \pi \varepsilon_{0}} \iint_{C} \frac{\sigma a d \theta d z}{\mathrm{PM}} \\
& =\frac{\sigma a}{4 \pi \varepsilon_{0}} \int_{0}^{2 \pi} \int_{-\frac{L}{2}}^{\frac{L}{2}} \frac{d \zeta}{\sqrt{r^{2}-2 \operatorname{arcos} \theta+a^{2}+(z-\zeta)^{2}}} d \theta \\
& =\frac{\sigma a}{4 \pi \varepsilon_{0}}\left[\int_{0}^{2 \pi} \operatorname{argsh}\left(\frac{z+\frac{L}{2}}{\sqrt{r^{2}-2 a r \cos \theta+a^{2}}}\right) d \theta-\int_{0}^{2 \pi} \operatorname{argsh}\left(\frac{z-\frac{L}{2}}{\sqrt{r^{2}-2 \operatorname{arcos} \theta+a^{2}}}\right) d \theta\right]
\end{aligned}
$$

where $\sigma$ is the surface charge density and $\varepsilon_{0}$ the dielectric permittivity of the void. The expression can be normalized as: 


$$
\tilde{V}\left(\frac{r}{a}, \frac{z}{a}\right)=\frac{4 \pi \varepsilon_{0}}{\sigma a} V(r, z)=\left[\int_{0}^{2 \pi} \operatorname{argsh}\left(\frac{\frac{z}{a}+\frac{1}{2} \frac{L}{a}}{\sqrt{\left(\frac{r}{a}\right)^{2}-2 \frac{r}{a} \cos \theta+1}}\right) d \theta-\int_{0}^{2 \pi} \operatorname{argsh}\left(\frac{\frac{z}{a}-\frac{1}{2} \frac{L}{a}}{\sqrt{\left(\frac{r}{a}\right)^{2}-2 \frac{r}{a} \cos \theta+1}}\right] d \theta\right]
$$

Finally, the application of the principle of the images gives the solution for a cylinder buried in a homogeneous half-space:

$$
\tilde{V}_{H S}\left(\frac{r}{a}, \frac{z}{a}\right)=\int_{0}^{2 \pi} \operatorname{argsh}\left(\frac{\frac{z}{a}+\frac{L}{a}}{\sqrt{\left(\frac{r}{a}\right)^{2}-2 \frac{r}{a} \cos \theta+1}}\right) d \theta-\int_{0}^{2 \pi} \operatorname{argsh}\left(\frac{\frac{z}{a}-\frac{L}{a}}{\sqrt{\left(\frac{r}{a}\right)^{2}-2 \frac{r}{a} \cos \theta+1}}\right) d \theta .
$$

Fig. A3 shows an example of such a solution.

\section{Annex 3. Validation of the discretization}

The system described in Annex I was simplified to implement the uniformly charged, hollow cylinder buried in a homogeneous space. In this case, indeed, Eq. (1) applies to the whole half-space, Eq. (2b) for the surface, Eqs. (3) at the infinite, and there is radial symmetry. The potential imposed to the cylinder, of radius $0.1 \mathrm{~m}$ and length $10 \mathrm{~m}$, was the solution determined in Annex II (Fig. A3b). The surface potential is decreasing (Fig. A4a), and the analytical and numerical solutions are very close, as evidences by the low values of the absolute difference (Fig. A4b) and of the relative differences (less than $0.6 \%$, Fig A4c). We are thus confident in the efficiency of the discretization scheme and the meshing that were used. 


\section{References}

Aarts, E.H.L., Van Laarhoven, P.J.M., 1985. Statistical cooling: a general approach to combinatorial optimization problems. Philips J. Res. 40, 193-226.

Bigalke, J., Grabner, E.W., 1997. The geobattery model: a contribution to large scale electrochemistry, Electrochim. Acta 42, 3443-3452.

Bogoslovsky, V.A., Ogilvy, A.A., 1973. Deformations of natural electric fields near drainage structures, Geophys. Prospect. 21, 716-723.

Bolève, A., Revil, A., Janod, F., Mattiuzo J.L., Jardani, A., 2007. Forward modelling and validation of a new formulation to compute self-potential signals associated with ground water flow, Hydrol. Earth Syst. Sci. 11, 1661-1671.

Castermant, J., Mendonça, C.A., Revil, A., Trolard, F., Bourrié, G., Linde N., 2008. Redox potential distribution inferred from self-potential measurements associated with the corrosion of a burden metallic body, Geophys. Prospect. 56, 269-282.

Darnet, M., Marquis, G., Sailhac, P., 2003. Estimating aquifer hydraulic properties from the inversion of surface streaming potential (SP) anomalies, Geophys. Res. Lett. 30 (13), 1679.

Jouniaux, L., Maineult, A., Naudet, V., Pessel, M., ailhac, P., 2009. Review of self-potential methods in hydrogeophysics, C. R. Geosci. 341, 928-936.

Patella, D., 1997. Introduction to ground surface self-potential tomography, Geophys. Prospect. $45,653-681$.

Petiau, G., 2000. Second generation of lead-lead chloride electrodes for geophysical applications, Pure. Appl. Geophys. 157, 357-382.

Petiau, G., Dupis, A., 1980. Noise, temperature coefficient, and long term stability of electrodes 
for telluric observations, Geophys. Prospect. 28, 792-804.

Revil, A., Naudet, V., Nouzaret, J., Pessel, M., 2003. Principles of electrography applied to selfpotential electrokientic sources and hydrological applications, Water Resources Res. 39 (5), 1114.

Revil, A., Karaoulis, M., Johnson, T., Kemna, A., 2012. Review: some low-frequency electrical methods for subsurface characterization and monitoring in hydrogeology, Hydrogeol. J. 20, 617-658.

Rittgers, J.M., Revil, A., Karaoulis, M., Mooney, M.A., Slater, L.D., Atekwana E.A., 2013. Selfpotential signals generated by the corrosion of buried metallic objects with application to contaminant plumes, Geophysics 78, EN65-EN82.

Rizzo, E., Suski, B., Revil, A., Straface, S., Troisi, S., 2004. Self-potential signals associated with pumping tests experiments, J. Geophys. Res. 109, B10203.

Sato, M., Mooney, H.M., 1960. The electrochemical mechanism of sulphide self-potentials, Geophysics 25, 226-249.

Stoll, J., Bigalke, J., Grabner, E.W., 1995. Electrochemical modelling of self-potential anomalies, Surv. Geophys. 16, 107-120.

Suski, B., Rizzo, E., Revil, A., 2004. A sandbox experiment of self-potential signals associated with a pumping test, Vadose Zone J. 3, 1193-1199.

Titov, K., Revil, A., Konosavsky, P., Straface, S., Troisi, S., 2005. Numerical modelling of selfpotential signals associated with a pumping test experiment, Geophys. J. Int. 162, 641650. 


\section{FIGURE CAPTIONS}

Fig. 1. Self-potential profile measured at the surface in the vicinity of the piezometer. Note that data were reduced from the mean value computed over the last eight points, in order to set the potential differences equal to zero far from the source.

Fig. 2. Model of the medium (radially symmetric). An unsatured zone of electrical conductivity $\sigma_{u}$ and thickness $W$ covers a zone of conductivity $\sigma_{s}$ saturated with a fluid of conductivity $\sigma_{f}$. The well of internal radius $R$ has a metallic casing of thickness $t$ until the depth $L_{m}$, then an impervious PVC casing until the depth $L_{m}+L_{p}$.

Fig. 3. Representation of the grid (irregular, rectangular mesh). a) large scale, b) domain of interest, c) near the well.

Fig. 4. Synthetic model. a) source (positions of the nodes of the spline: 0, 1, 4.75, 9.5 and $11.1 \mathrm{~m}$; associated potential values: $-0.2,-0.21,-0.6,0.8$ an $0 \mathrm{~V}$ respectively), b) resulting potential distribution in the vicinity of the well, c) resulting self-potential profile at the surface.

Fig. 5. Simplified flow chart of the simulated annealing algorithm. Explanations in the text.

Fig. 6. Example of evolution of key outputs of the simulated annealing algorithm. a) temperature, b) cost-function ( $\mathrm{L}_{1}$-norm of the difference between the prediction and data), c) number of models per iterations and d) deduced rejection rate. In b), the red line corresponds to the global minimum value of the $\mathrm{L}_{1}$-norm (i.e., minimum value over all already performed iterations), the blue line to the minimum value of the $\mathrm{L}_{1}$-norm for each iteration, and the purple line to the maximum value of the $\mathrm{L}_{1}$-norm for each iteration. Inversion of the synthetic data shown in Fig. 4c, using a spline with six nodes linearly positioned with depth and starting from $\left[\begin{array}{llllll}0 & 0 & 0 & 0 & 0 & 0\end{array}\right] \mathrm{V}$, the potential value of the deepest node being kept 
fixed.

Fig. 7. Example of convergence of the algorithm. a) evolution of the potential value at a node for a first inversion starting from $0, b$ ) associated values of the potential at this node for all accepted models with respect to their $\mathrm{L}_{1}$-norm values, with $\mathrm{c}$ ) zoom on the lower $\mathrm{L}_{1}$-norm values, and d) distribution of the potential values having a $\mathrm{L}_{1}$-norm less than 2.2. In this case, the convergence cannot be considered as achieved since the optimal, average and median values are not equal. Same graphics for a final inversion (e, f, g, h). In this case, the convergence can be considered as achieved since the solution is stable and the optimal, average and median values of the histogram are very close.

Fig. 8. Example of inversion of the synthetic data (Fig. 4c). a) retrieved potential distributions on the metallic casing using splines with 4, 5, 6 and 7 nodes linearly positioned with depth, the potential value of the deepest node being kept fixed to 0 , and $b$ ) resulting potential profiles at the surface. Fitting values $\left(\mathrm{L}_{1}\right.$-norm, $\mathrm{L}_{2}$-norm and adjustment coefficient $\left.r^{2}\right)$ are computed for the well potential (since the true solution is known, grey thick line) and for the surface potential (in this case the $\mathrm{L}_{1}$-norm is the parameter effectively governing the inversion). All retrieved source can explain the data satisfactorily, but only the solutions with 6 and 7 nodes correspond to the input source.

Fig. 9. Example of inversion of the synthetic data (Fig. 4c): retrieved potential distributions on the metallic casing using splines with 4, 5, 6 and 7 nodes linearly positioned with depth, the value of the deepest node being floating like the others. Only the solutions with 6 and 7 nodes are similar to the input source (grey thick line).

Fig. 10. Example of inversion of the synthetic data with noise: retrieved potential distributions (a) on the casing with added Gaussian noise (mean $0 \mathrm{mV}$, standard-deviation $2 \mathrm{mV}$, b), using a spleen with 5 nodes positioned as those used in the generation of the synthetic source 
(Fig. 4a). The retrieve source deviates slighlty from the true source (grey thick line).

Fig. 11. Inversion of the field data (Fig. 1). a) retrieved potential distributions on the casing using splines with 4, 5, 6 and 7 nodes linearly positionned with depth, the value of the deepest node being floating like the others; b) resulting potential profiles at the surface. Solutions with 5, 6 and 7 nodes are roughly similar, and evidence a dipolar distribution of potential along the metallic casing.

Fig. 12. Estimation of the potential distribution generating the field data (6-node spline solution, Fig. 10a).

Fig. A1. Discretization scheme and notations for the finite-difference.

Fig. A2. Geometry of the hollow cylinder and notations.

Fig. A3. Distribution of the normalized potential for a buried, finite, hollow and uniformly charged cylinder of radius $a$ and length $L=4 a$ (a) and normalized potential distribution along the external wall of the cylinder (b).

Fig. A4. Validation of the implementation for the direct problem on the buried, finite, hollow and uniformly charged cylinder. a) potential profile at the surface, b) difference between computed potential and analytical solution, c) relative difference. The low values of the differences prove that the discretization of the space and the used finite-difference scheme that are acceptable. 
Figure 1

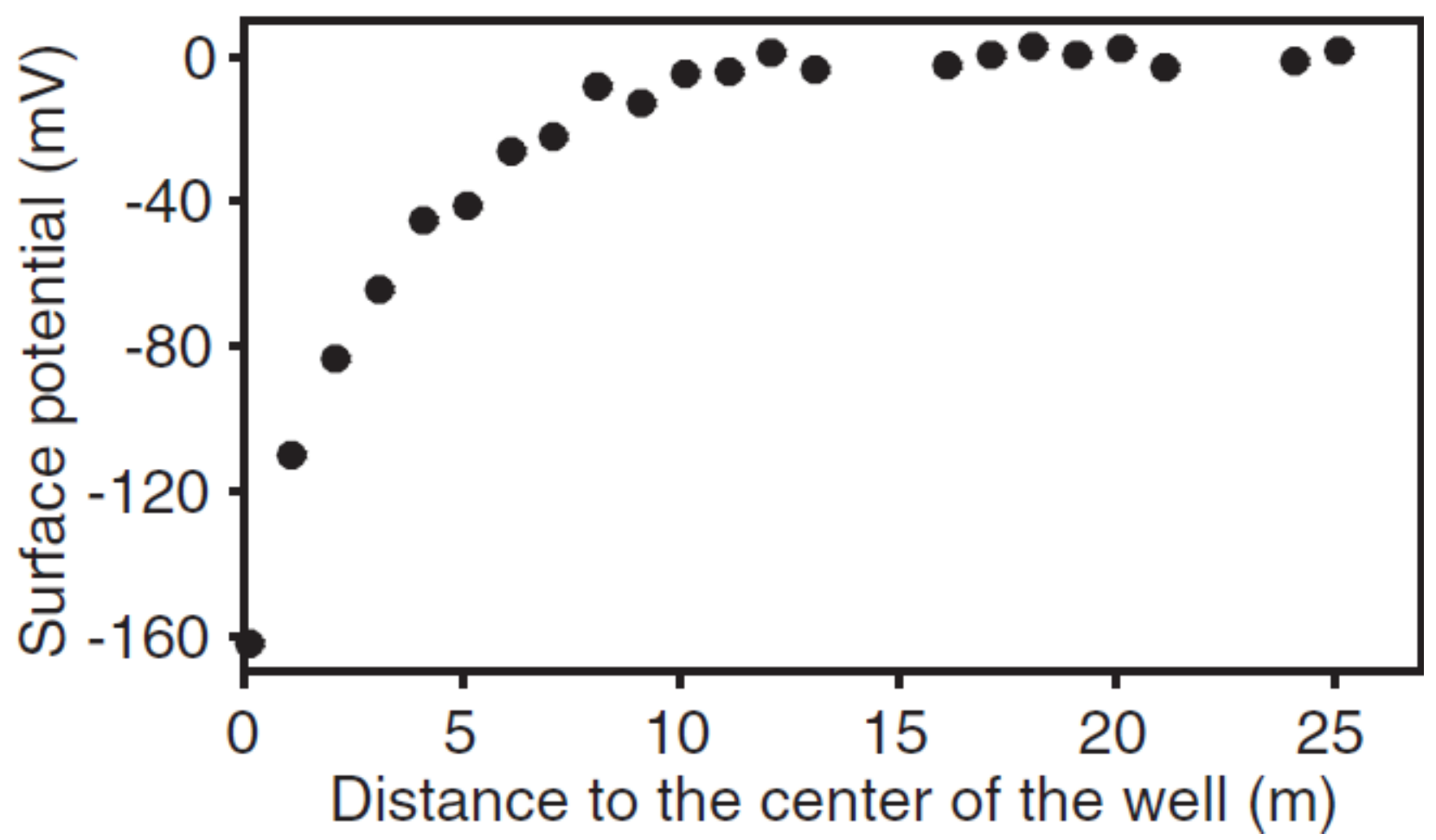

Figure 1 
Flgure 2

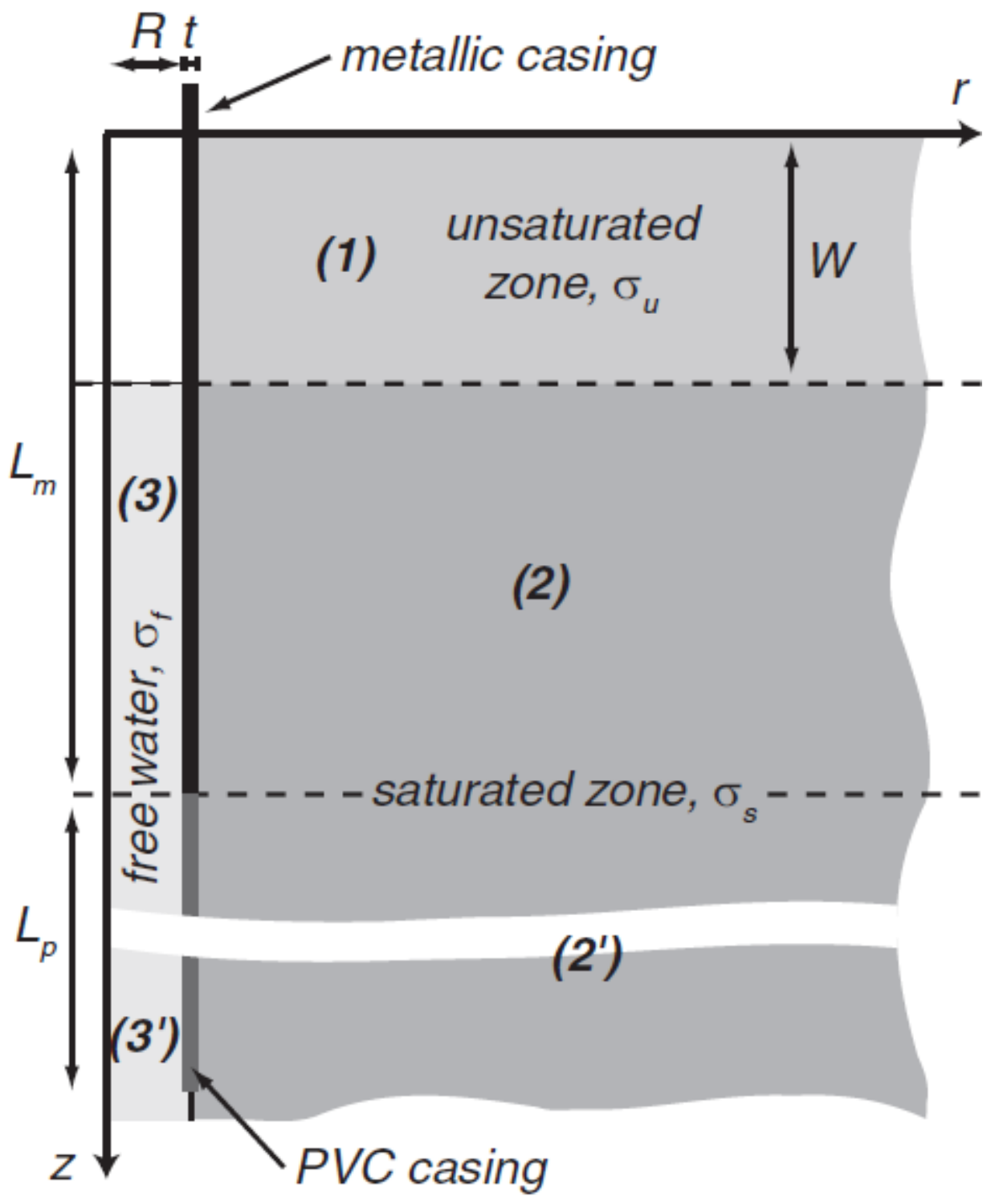

Figure 2 


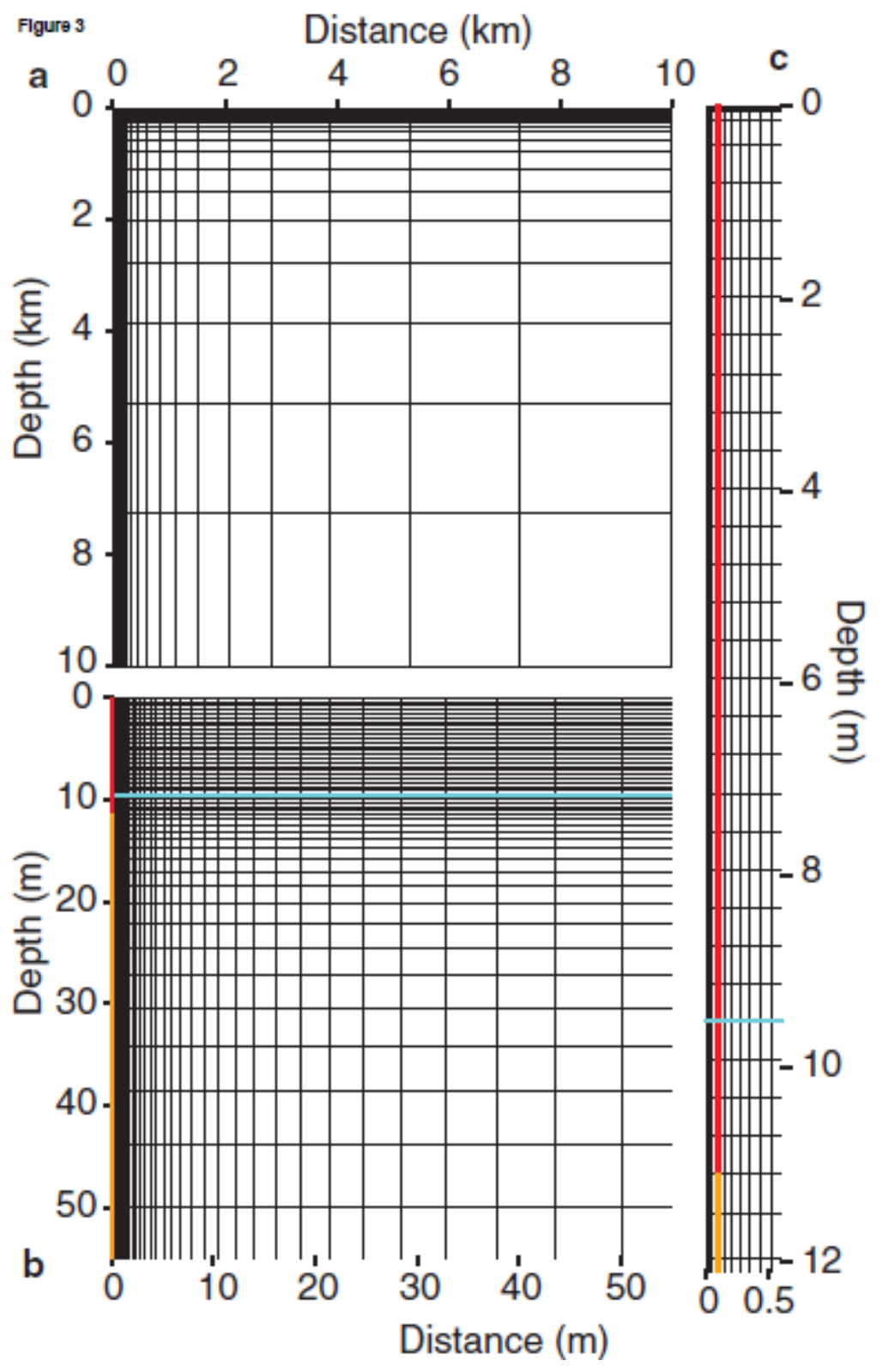

Figure 3 
Flgure 4
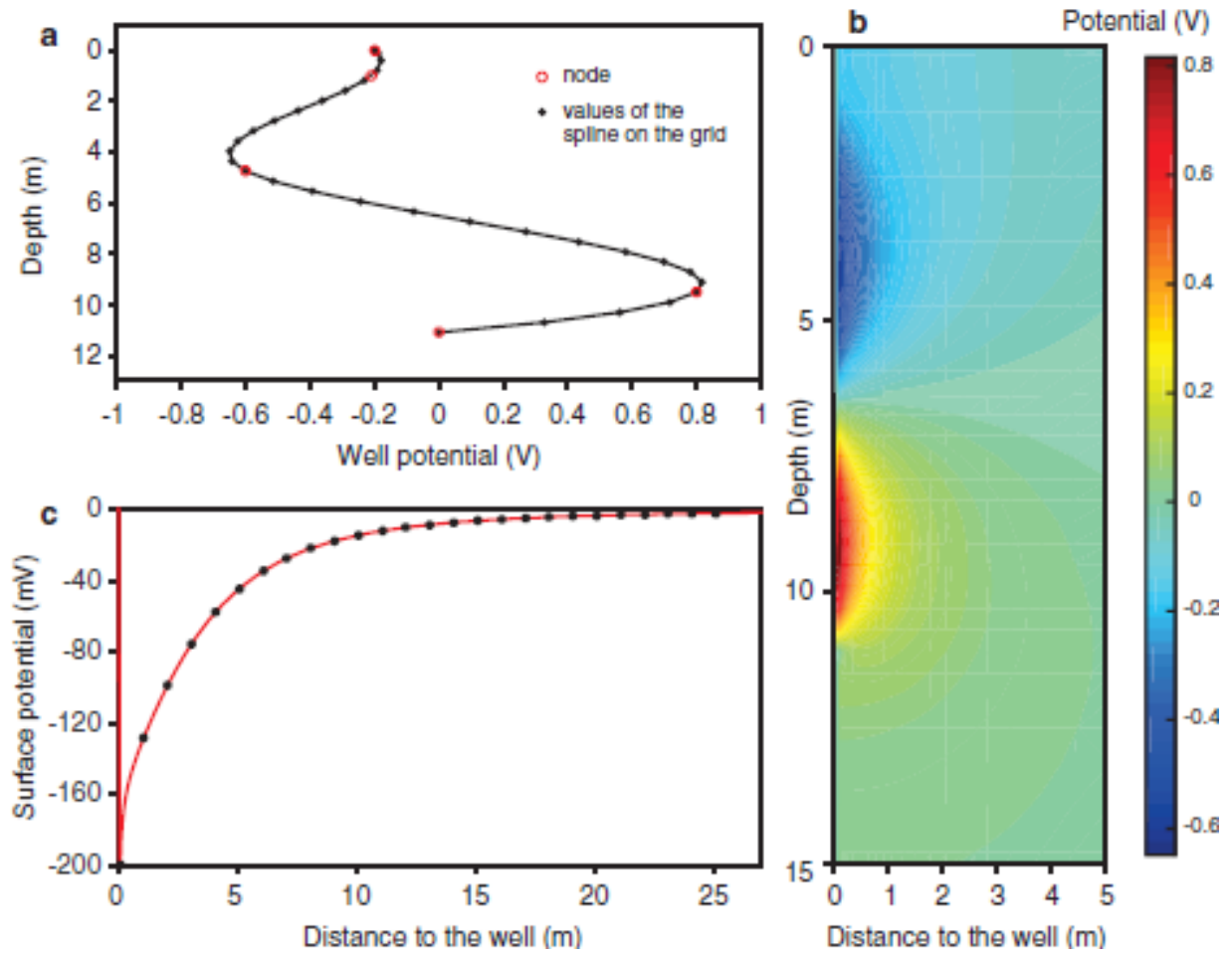

Figure 4 


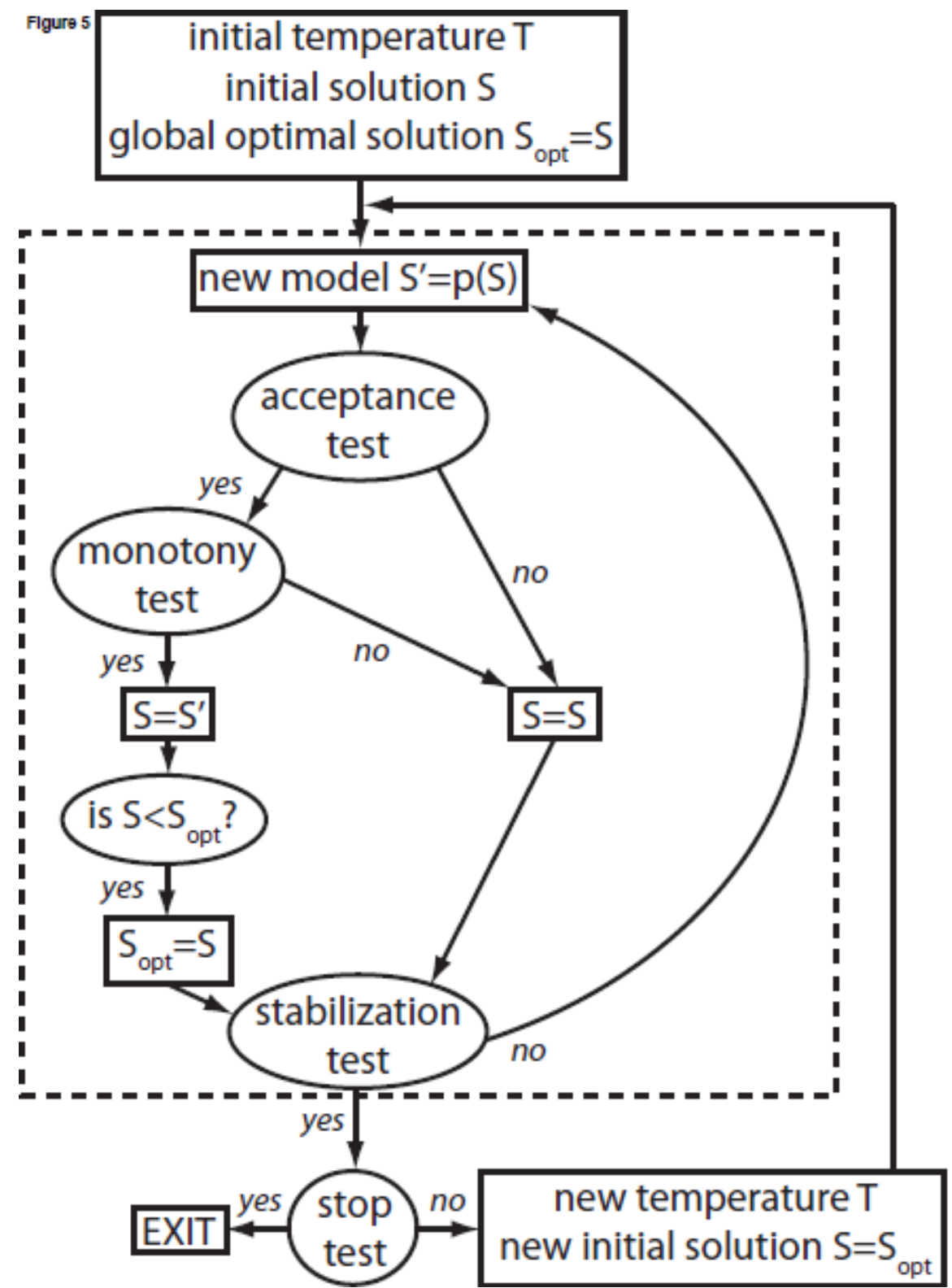

Figure 5 
Figure 6
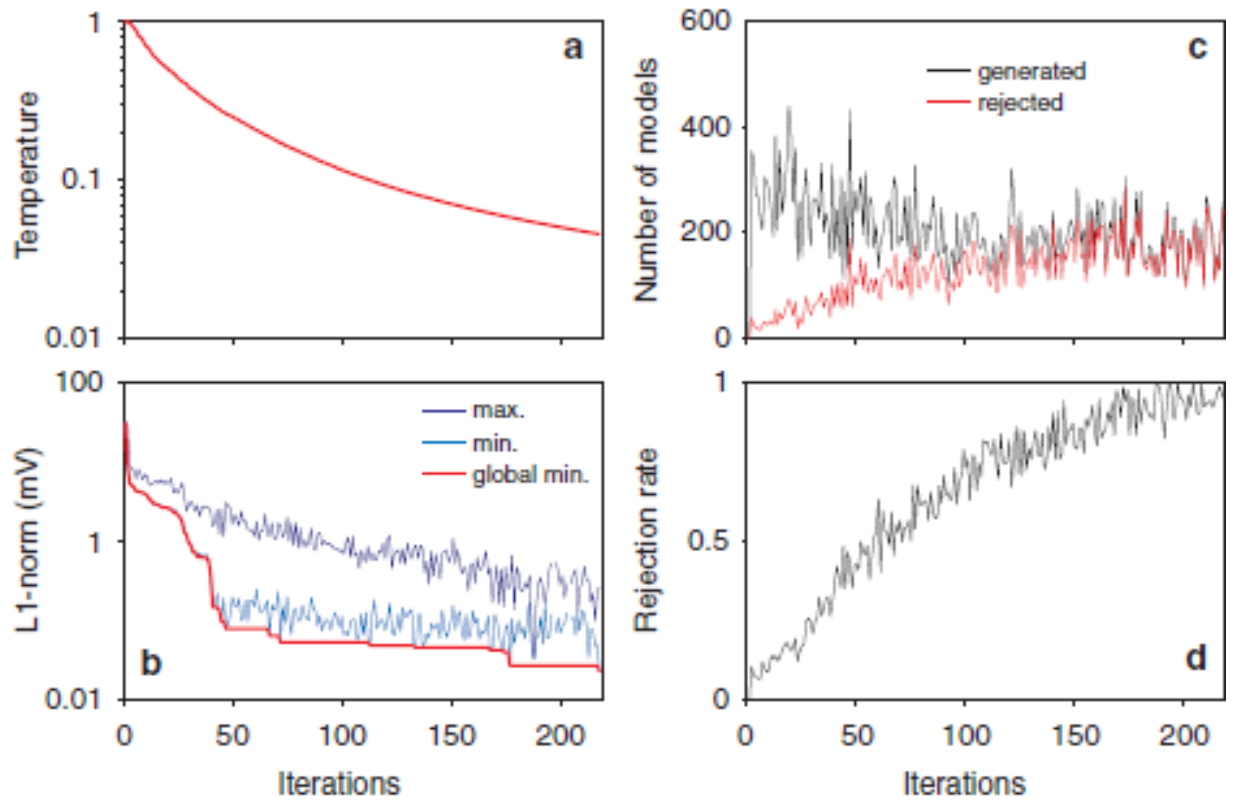

Figure 6 

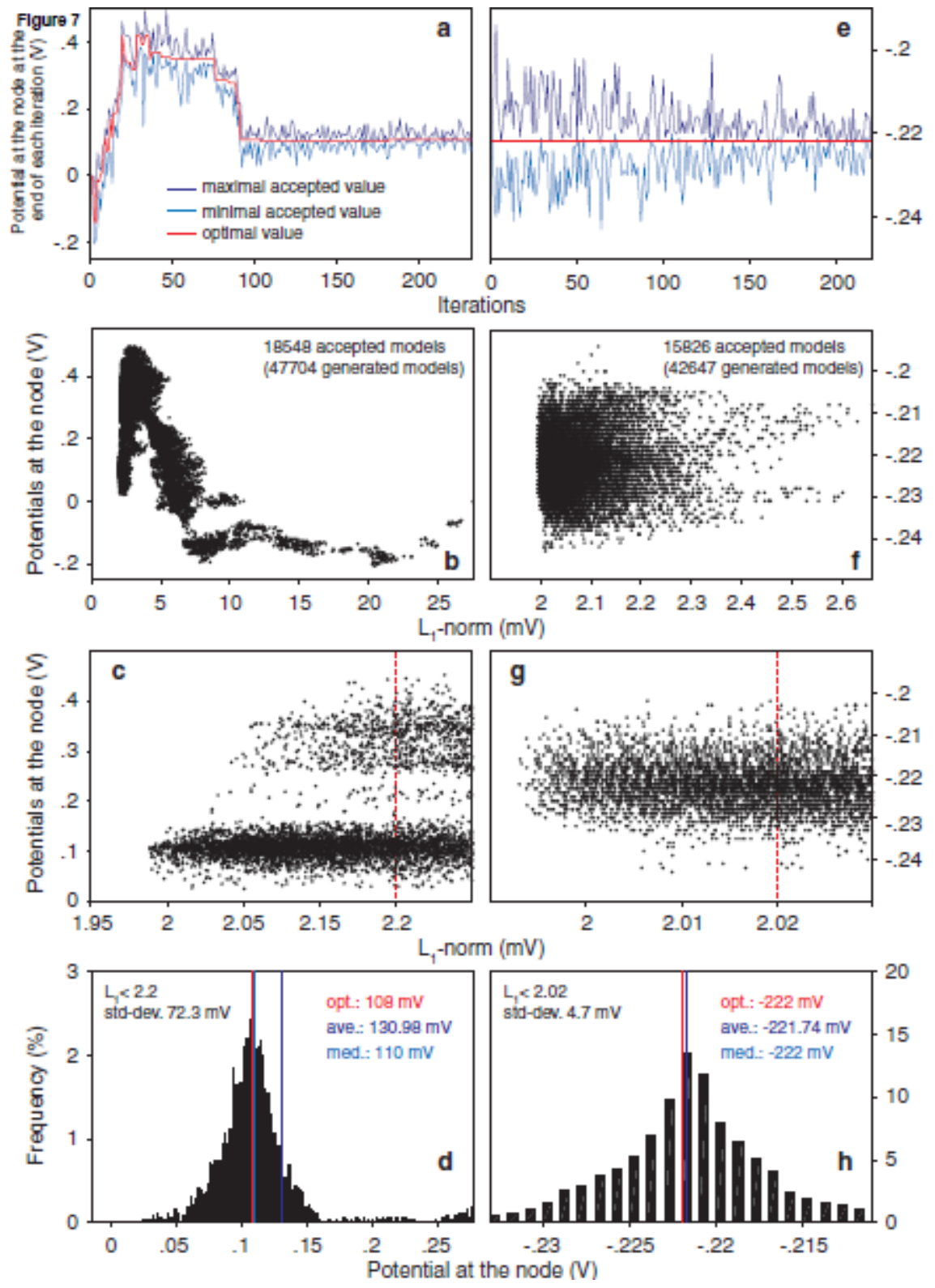

Figure 7 
Figure 8
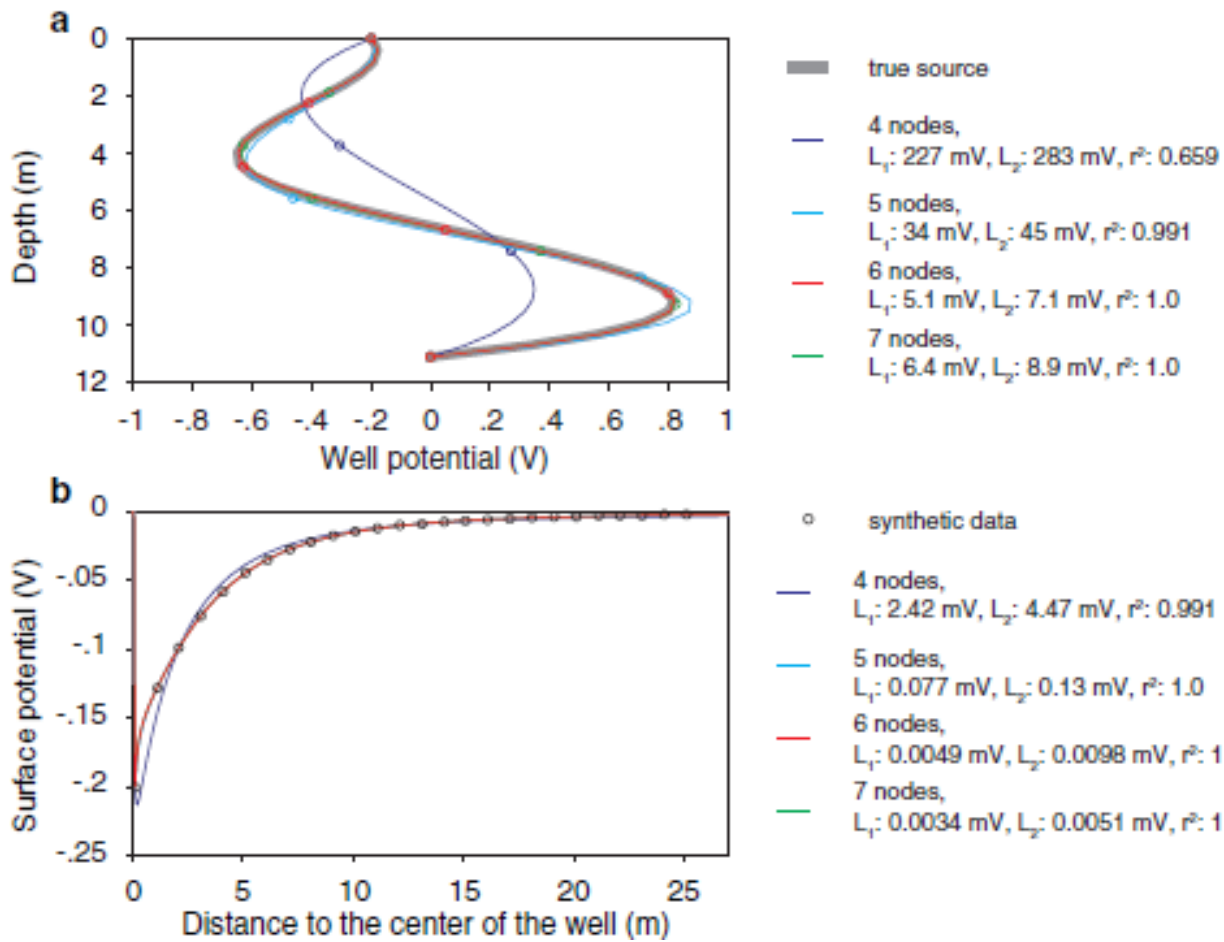

- synthetic data

- 4 nodes,

$L_{1}=2.42 \mathrm{mV}, L_{z}: 4.47 \mathrm{mV}, \mathrm{r}^{2}: 0.991$

5 nodes, $L_{1}: 0.077 \mathrm{mV}, L_{2}: 0.13 \mathrm{mV}, r^{2}: 1.0$ 6 nodes,

- $\quad L_{1}=0.0049 \mathrm{mV}, L_{2}=0.0098 \mathrm{mV}, r^{2}: 1$

7 nodes,

- $L_{1}=0.0034 \mathrm{mV}, L_{2}=0.0051 \mathrm{mV}, r^{2}: 1$

Figure 8 
Figure 9

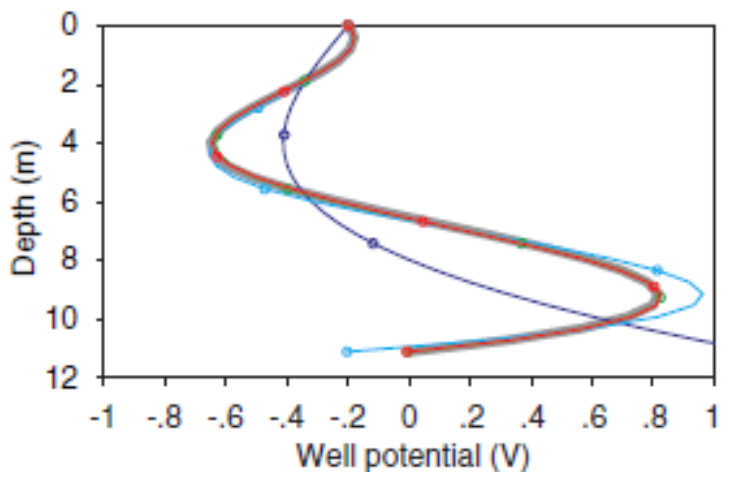

true source

4 nodes,

$L_{1}: 245 \mathrm{mV}, L_{2}: 351 \mathrm{mV}, r^{2}: 0.476$

5 nodes,

$\mathrm{L}_{\mathrm{i}}: 48 \mathrm{mV}, \mathrm{L}_{\mathrm{z}}: 72 \mathrm{mV}, \mathrm{r}^{2}: 0.978$

- 6 nodes,

$\mathrm{L}_{\mathrm{\gamma}}: 6.1 \mathrm{mV}, \mathrm{L}_{2}: 8.4 \mathrm{mV}, \mathrm{r}^{2}: 1.0$

7 nodes,

- $\quad L_{\mathrm{s}}: 6.6 \mathrm{mV}, \mathrm{L}_{\mathrm{z}}: 9.0 \mathrm{mV}, \mathrm{r}^{2}: 1.0$

Figure 9 
Figure 10
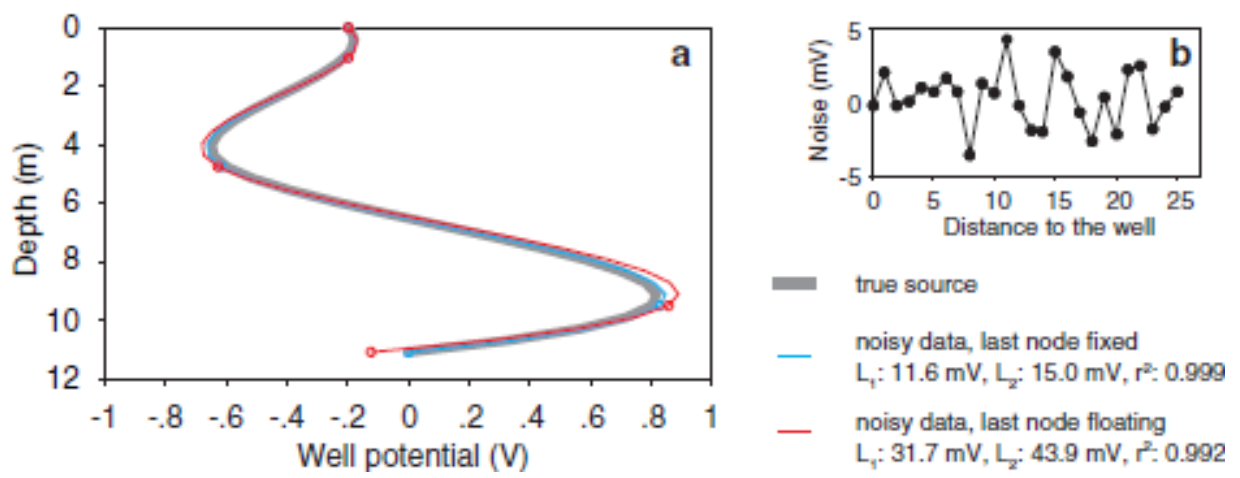

Figure 10 
Figure 11
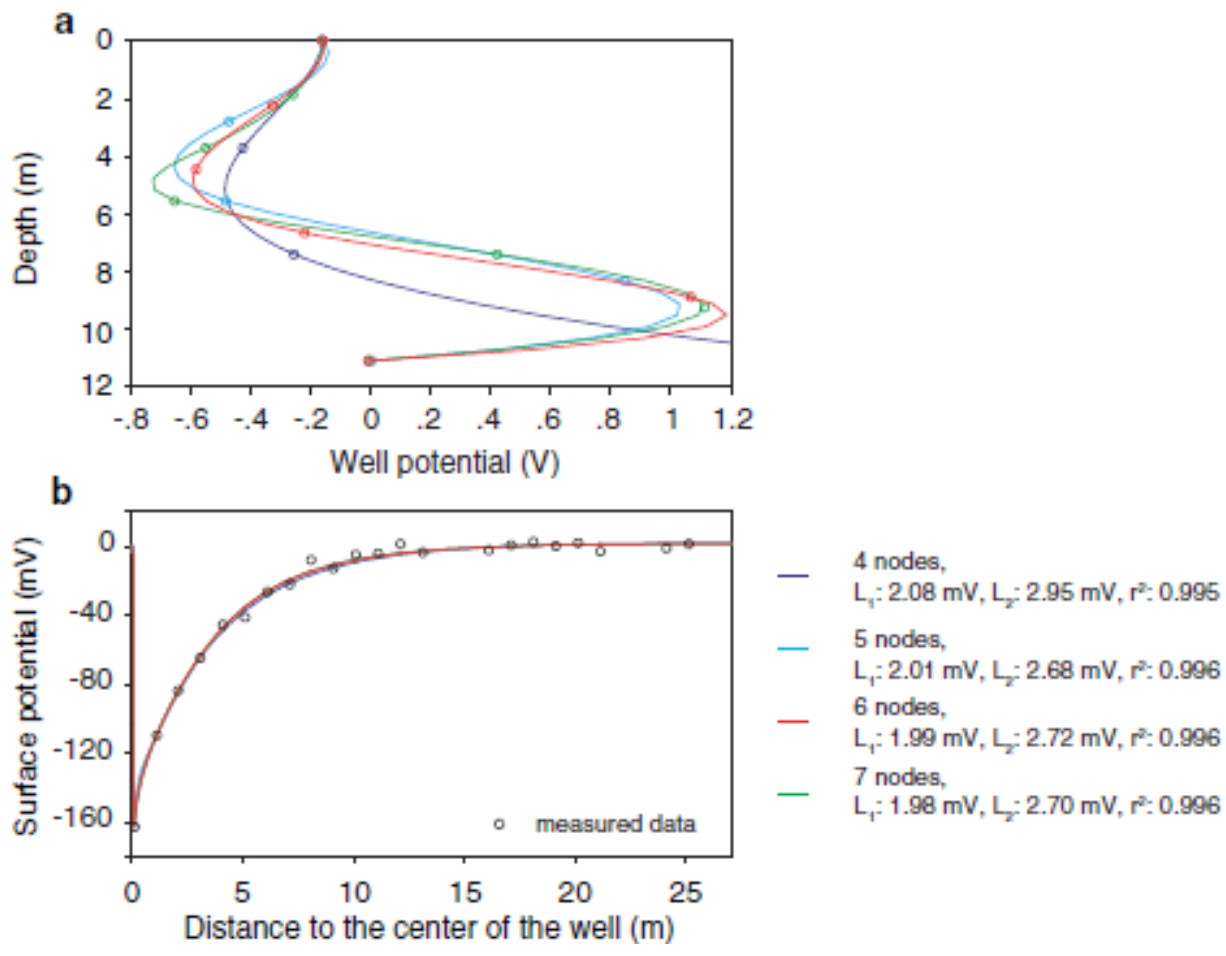

Figure 11 


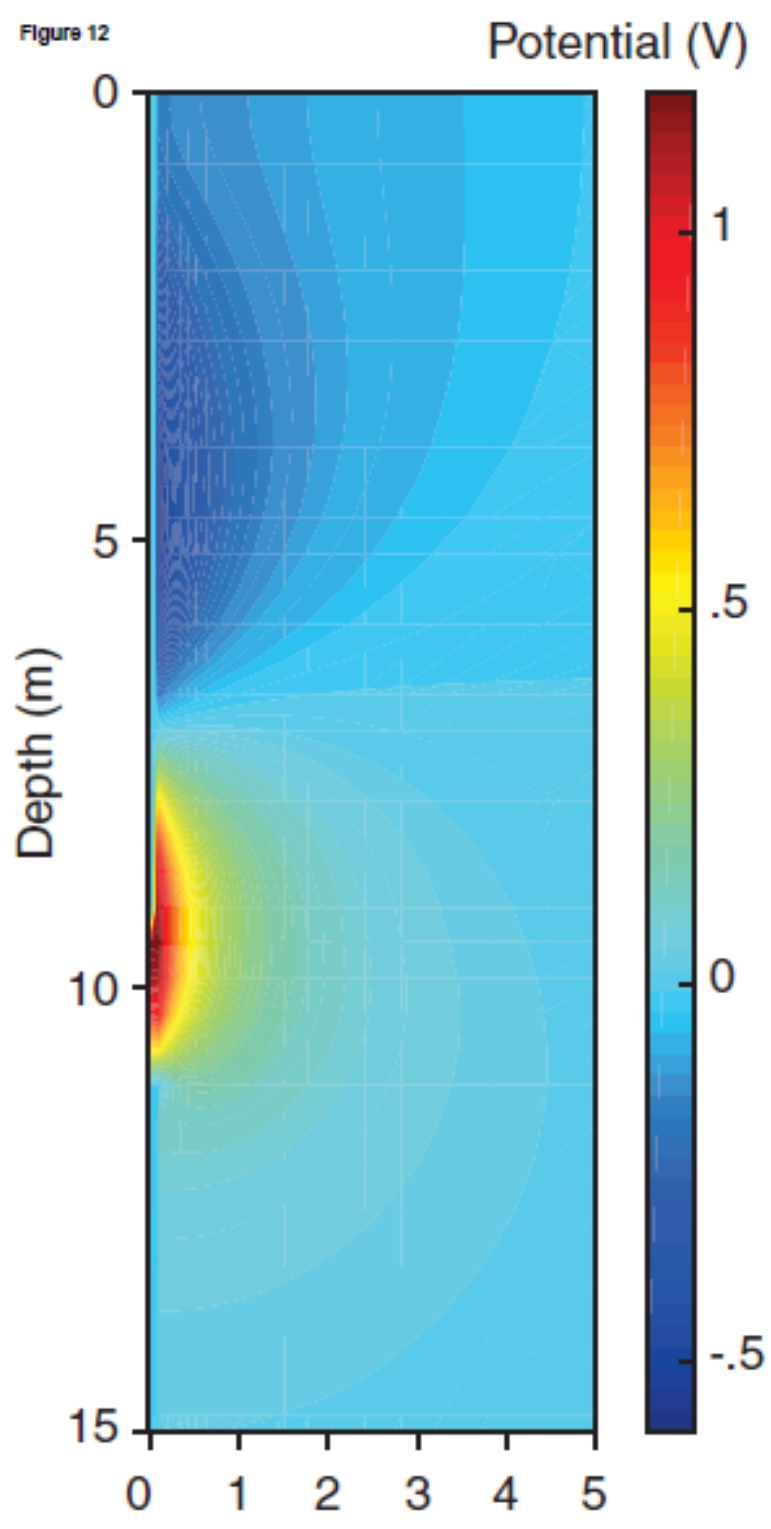

Distance to the well $(\mathrm{m})$

Figure 12 
Figure A1

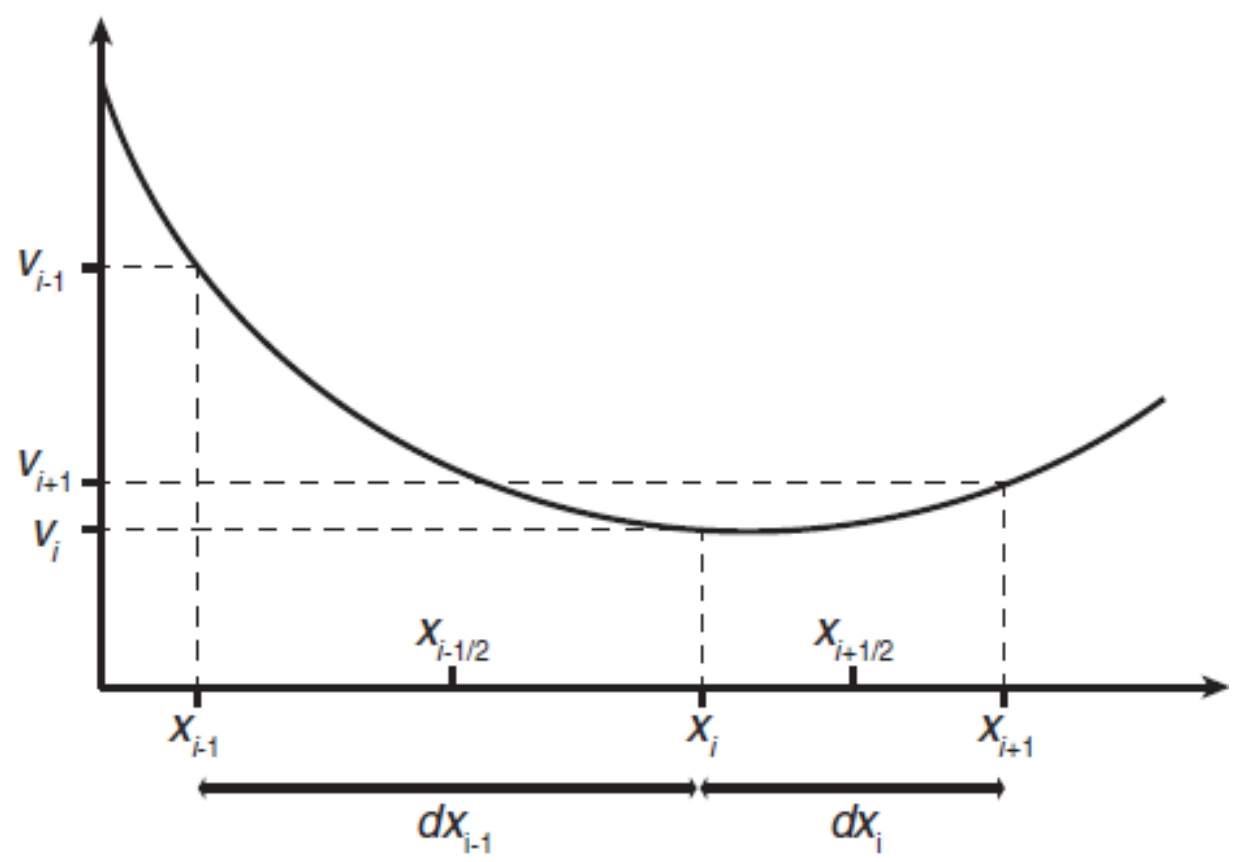

Figure A1 
Flgure A2

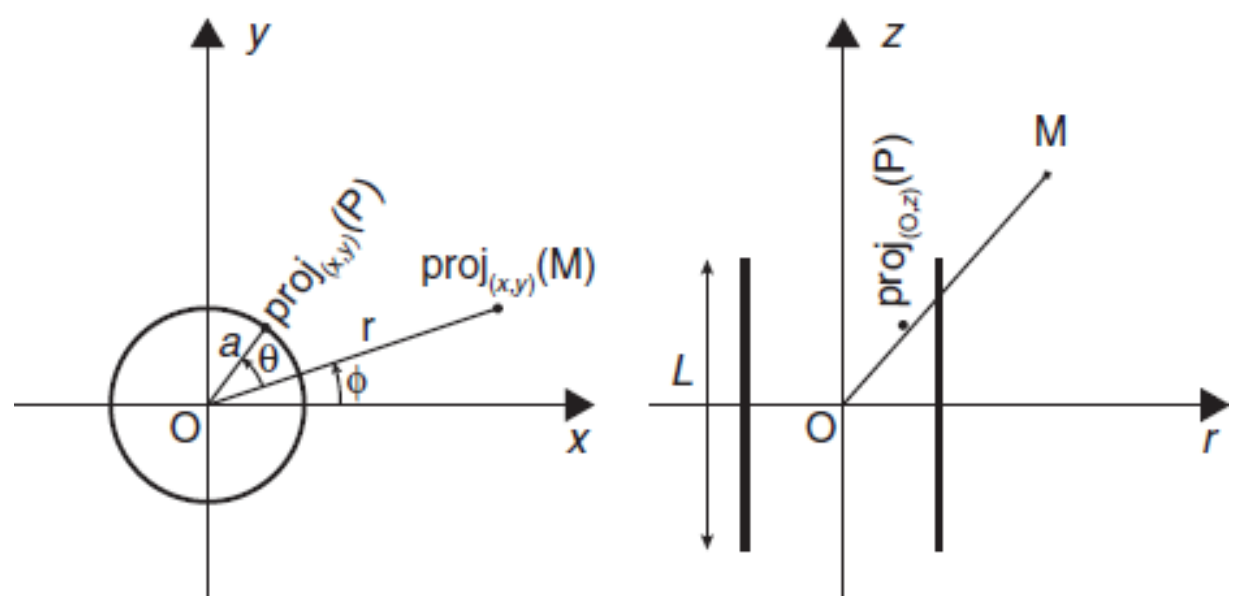

Figure A2 
Figure A3

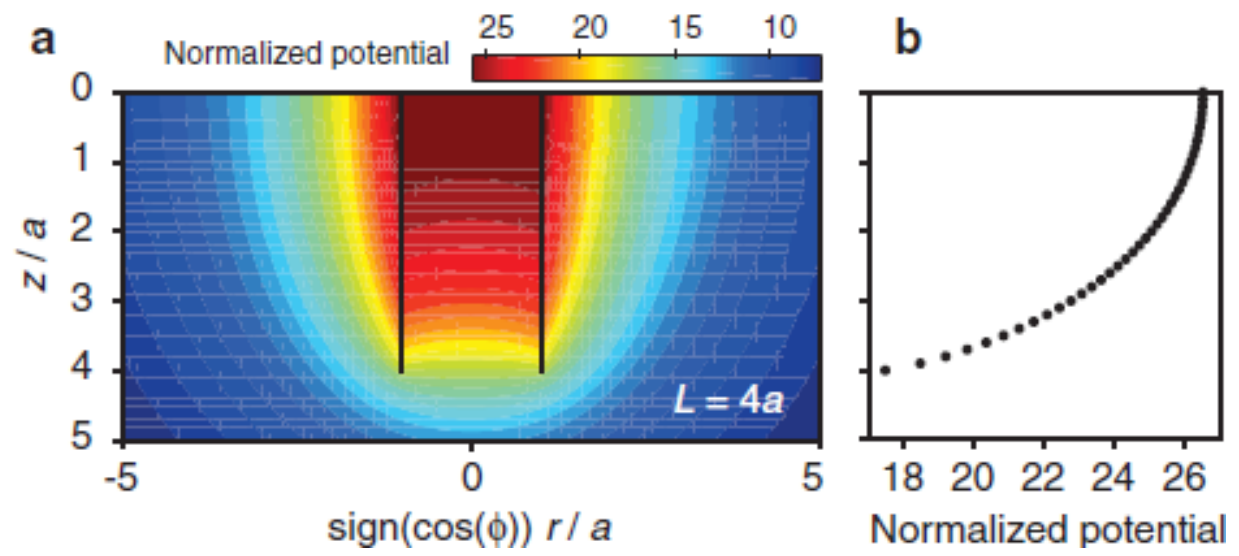

Figure A3 

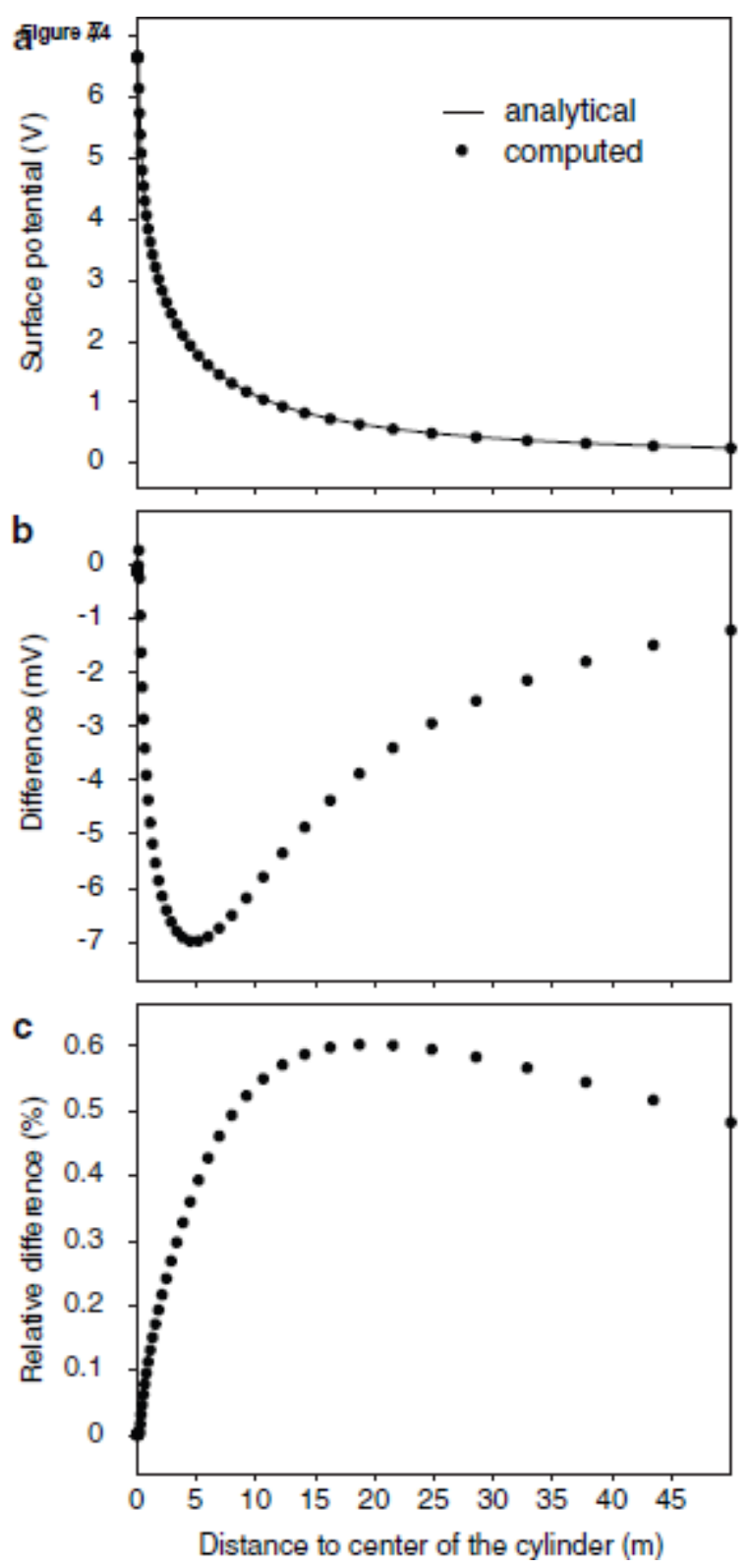

Figure A4 
Highlights

- A non-deterministic procedure was designed to invert SP surface profile in terms of potential distribution along metallic casing.

- A real field case was inverted.

- The determined potential distribution is coherent with laboratory results from the literature. 